\title{
Theory of deeply virtual Compton scattering off the unpolarized proton
}

\author{
Brandon Kriesten ${ }^{*}$ and Simonetta Liuti $\odot^{\dagger}$ \\ Department of Physics, University of Virginia, Charlottesville, Virginia 22904, USA
}

(Received 4 November 2020; accepted 24 November 2021; published 18 January 2022)

\begin{abstract}
Using the helicity amplitudes formalism, we study deeply virtual exclusive electron photoproduction off an unpolarized nucleon target, $e p \rightarrow e^{\prime} p^{\prime} \gamma$, through a range of kinematics both in the fixed target setting with initial electron energies of 6,11 , and $24 \mathrm{GeV}$ and for an electron ion collider. We reformulate the cross section bringing to the forefront the defining features of the $e p \rightarrow e^{\prime} p^{\prime} \gamma$ process, where the observables are expressed as bilinear products of the independent helicity amplitudes which completely describe it in terms of the electric, magnetic, and axial currents of the nucleon. These contributions are checked against the Fourier harmonics-based formalism which has provided so far the underlying mathematical framework to study deeply virtual Compton scattering (DVCS) and related experiments. Using theoretical model calculations of the twist-two generalized parton distributions, $H, E, \widetilde{H}$, and $\widetilde{E}$, we uncover large discrepancies between the harmonic series and our proposed framework. Most importantly, these numerical differences appear in the intermediate $Q^{2}$ range which represents a sweet spot for extracting generalized parton distributions from data. We provide a framework that is ideal, on one side, to study and compare the different conventions that can be used to describe the leading order contribution to DVCS in QCD, while on the other it facilitates a quantitative extraction of physically meaningful information from experiment through traceable and controllable approximations in the intermediate $Q^{2}$ region.
\end{abstract}

DOI: 10.1103/PhysRevD.105.016015

\section{INTRODUCTION}

Deeply virtual Compton scattering (DVCS) is measured through the exclusive process, $e p \rightarrow e^{\prime} p^{\prime} \gamma$, where, in the one photon exchange approximation, the virtual photon four-momenta squared, $Q^{2}$, provides a hard scale for the process. Quantum chromodynamics (QCD) factorization theorems allow us to single out the perturbative, short distance reaction from the nonperturbative, long distance matrix elements described in terms of generalized parton distributions (GPDs) [1,2]. ${ }^{1}$ GPDs encode new information on the internal dynamics of the proton that will allow us, through a combined analysis of experimental data and lattice QCD results, to ultimately map out its 3D structure. These distributions enter the observables embedded in the Compton form factors (CFFs), which are convolutions over the longitudinal momentum variable $x$ with complex QCD Wilson coefficient functions (see reviews in [6-8]).

\footnotetext{
*btk8bh@virginia.edu

†s14y@virginia.edu

${ }^{1}$ Detailed proofs of factorization for deeply virtual exclusive processes can be found in [3-5].

Published by the American Physical Society under the terms of the Creative Commons Attribution 4.0 International license. Further distribution of this work must maintain attribution to the author(s) and the published article's title, journal citation, and DOI. Funded by SCOAP ${ }^{3}$.
}

At leading order, four quark chirality conserving GPDs giving eight CFFs describe all possible quark $\left(P_{q}\right)$ proton $\left(P_{p}\right)$ polarization configurations, $P_{q} P_{p}=U U, L L, U T, L T$, allowed by parity conservation, time reversal invariance, and charge conjugation. The eight CFFs appear simultaneously in all of the deeply virtual exclusive scattering experimental observables, independent of the specific beam-target polarization configuration. This poses a challenge for the extraction of CFFs from experiments which are affected by large theoretical uncertainties (see [8] for a detailed list of experiments).

In Ref. [9], we introduced a new theoretical formulation of the cross section for the $e p \rightarrow e^{\prime} p^{\prime} \gamma$ process in all polarization configurations for the incoming electron and proton target. The main goal of Ref. [9] was to provide a formulation of the cross section in terms of CFFs that allowed one to evaluate precisely the impact of order $1 / Q$ and higher power corrections of kinematic and dynamical origins.

Focusing on the unpolarized target case, in this paper, we illustrate how the complete calculation of the cross section leads to a more direct interpretation in terms of the electric, magnetic, and axial current contributions to DVCS. In our approach, the Bethe-Heitler (BH)-DVCS interference term of the $e p \rightarrow e^{\prime} p^{\prime} \gamma$ cross section exhibits a structure analogous to the cross section for $e p$ elastic scattering (see, e.g., Refs. [10,11] and references therein), preserving the structure of the nucleon charge, magnetic, 
and axial current contributions to the cross section from the underlying helicity amplitude configurations $[12,13]$.

Previous studies, referring, in particular, to the widely adopted formalism of Refs. [7,14-17] Belitsky-KirchnerMuller (BKM) are organized, instead, in terms of harmonics of the azimuthal angle, $\phi$, and in kinematic powers of $1 / Q$. On one side, the harmonics-based formalism presents the appealing aspect of associating a dominant harmonic for each observable, therefore providing a simplified framework for experimental measurements. While this approach was perhaps needed in the pioneering analyses of the HERMES era [18], at present, with Jefferson Lab @ $12 \mathrm{GeV}$ and the future Electron Ion Collider (EIC) we are now entering a precision era where information on the physics content of the deeply virtual cross section, in particular, its electroweak structure, can be studied. This structure, in turn, bears important consequences for building a phenomenological framework to quantitatively study the composition of angular momentum, mass, and other mechanical properties of the proton in terms of quark and gluon contributions.

Our new framework radically changes the extraction of CFFs from data [19]. In Refs. [15,16], according to the harmonics expansion, the magnetic contribution containing the angular momentum related CFF combination $(H+E)$ to the BH-DVCS interference term was deemed as power suppressed and, therefore, not included in the leading order formula. Following Ref. [19], we introduce linear fits of the DVCS data that enable us to extract the magnetic contribution for the first time, with a relatively small size error.

While our approach shares a common starting point with the initial studies of the cross section performed in Refs. [20-22], our results are in line with the recent study in Refs. [23-25], broadly labeled as "finite $t$ and target mass corrections", in that we recognize the importance of kinematic contributions originating from the choice of reference frames where the QCD hadronic tensor and $\mathrm{BH}$ proton current are evaluated.

A further advantage of the new formalism is that it provides compact expressions for the various kinematic coefficients expressed in terms of invariant four-vector products. From the practical point of view, we provide simplified expressions that can be readily used in the development of simulations and pseudodata, for both fixed target and collider settings. A striking example is given by the form of the $\mathrm{BH}$ unpolarized cross section which reduces, in our case, to two lines of computation. ${ }^{2}$ The DVCS contribution is organized in terms of structure functions for the various polarization configurations for the lepton beam and nucleon target, in line with the general cross section formulations of Refs. [13,26].

\footnotetext{
${ }^{2}$ Our results are consistent with a previous calculation for the unpolarized $\mathrm{BH}$ cross section given in terms of Mandelstam invariants Refs. [20-22].
}

The work presented in this paper is organized in the following points:

(i) We discuss in detail the physics content of the expressions derived in Ref. [9], including the origin of the phase dependence and the polarization configurations for both the twist-two and twist-three contributions (Sec. II).

(ii) We perform a detailed numerical comparison with the formalism of BKM for the unpolarized cross section including the $\mathrm{BH}, \mathrm{DVCS}$, and BH-DVCS interference terms. The comparison is valid up to twist three, using the same model calculation of the GPDs for both the present paper's and the BKM expressions. This ensures that the differences can be ascribed entirely to the formalism, (Sec. III).

(iii) We cover a range of kinematic regions, from Jefferson Lab@6 GeV and @12 GeV to a hypothetical energy value of $24 \mathrm{GeV}$ fixed target configuration [27] to the EIcC [28] and EIC [29]. All graphs are shown and discussed in Sec. III.

Finally, we write our conclusions and outlook in Sec. IV.

\section{UNPOLARIZED SCATTERING CROSS SECTION}

The cross section for the deeply virtual photon electroproduction process, $e(k)+p \rightarrow e^{\prime}\left(k^{\prime}\right)+p^{\prime}+\gamma^{\prime}\left(q^{\prime}\right)$, on an unpolarized proton, is derived from a coherent superposition of the DVCS and BH amplitudes, where the $\mathrm{BH}$ contribution arises when the final photon is emitted from either the initial or final electron. It is therefore a competing mechanism to the DVCS process evaluated at low momentum transfer, $-t \ll Q^{2}$. One has

$$
\begin{aligned}
\frac{d^{5} \sigma}{d x_{B j} d Q^{2} d|t| d \phi} & =\Gamma|T|^{2}=\Gamma\left|T_{\mathrm{BH}}+T_{\mathrm{DVCS}}\right|^{2} \\
& =\Gamma\left(\left|T_{\mathrm{BH}}\right|^{2}+\left|T_{\mathrm{DVCS}}\right|^{2}+\mathcal{I}\right),
\end{aligned}
$$

with the interference term, $\mathcal{I}$, being defined as

$$
\mathcal{I}=T_{\mathrm{BH}}^{*} T_{\mathrm{DVCS}}+T_{\mathrm{DVCS}}^{*} T_{\mathrm{BH}},
$$

where $\Gamma$ is the flux factor,

$$
\Gamma=\frac{\alpha^{3}}{16 \pi^{2}\left(s-M^{2}\right)^{2} \sqrt{1+\gamma^{2}} x_{B j}},
$$

where $\alpha$ is the electromagnetic fine structure constant. We define the relativistic invariants, $Q^{2}=-\left(k-k^{\prime}\right)^{2}$, $t=\Delta^{2}=\left(p^{\prime}-p\right)^{2}, x_{B j}=Q^{2} / 2(p q)$, with $\nu=(p q) / M$, $s=(p+k)^{2} ; \phi$ is the angle between the lepton and hadron planes and $M$ the proton mass. 
The amplitude for the DVCS process reads

$$
T_{\mathrm{DVCS}}=e^{3} j_{\mathrm{DVCS}}^{\mu} \frac{\widetilde{g}_{\mu \nu}}{q^{2}} J_{\mathrm{DVCS}}^{\nu}
$$

where the lepton and hadron currents are, respectively, given by

$$
\begin{aligned}
& j_{\mathrm{DVCS}}^{\mu}=\bar{u}\left(k^{\prime}, h\right) \gamma^{\mu} u(k, h), \\
& J_{\mathrm{DVCS}}^{\nu}=\mathcal{W}^{\mu \nu}\left(p, p^{\prime}\right)\left(\varepsilon_{\mu}^{\Lambda_{\gamma^{\prime}}}\left(q^{\prime}\right)\right)^{*},
\end{aligned}
$$

where $W_{\mu \nu}$ is the DVCS hadronic tensor, and $\varepsilon^{\Lambda_{\gamma^{\prime} \mu}}\left(q^{\prime}\right)$ is the polarization vector of the outgoing photon, $\gamma^{\prime}$. For BH one has

$$
T_{\mathrm{BH}}=\frac{e^{3}}{\Delta^{2}} j_{\mathrm{BH}}^{\mu}\left(J_{\mathrm{BH}}\right)_{\mu},
$$

with

$$
\begin{aligned}
& j_{\mathrm{BH}}^{\mu}=\left(\varepsilon^{\Lambda_{\gamma^{\prime}} \nu}\left(q^{\prime}\right)\right)^{*} L_{\mu \nu}^{h}\left(k, k^{\prime}, q^{\prime}\right), \\
& \left(J_{\mathrm{BH}}\right)_{\mu}=\bar{U}\left(p^{\prime}, \Lambda^{\prime}\right) \Gamma_{\mu} U(p, \Lambda),
\end{aligned}
$$

where $L_{\mu \nu}^{h}$ is the tensor for electron scattering off the proton with the emission of a final photon, and $\Gamma_{\mu}$ is the usual current operator given in terms of the Dirac and Pauli form factors, $F_{1}$ and $F_{2}$, as

$$
\Gamma_{\mu}=\left(F_{1}+F_{2}\right) \gamma_{\mu}-\frac{\left(p+p^{\prime}\right)_{\mu}}{2 M} F_{2} .
$$

The DVCS amplitude involves the photon projection operator, $\widetilde{g}_{\mu \nu}$, which is defined by the expansion [30,31]

$$
\widetilde{g}_{\mu \nu}=\sum_{\Lambda_{\gamma^{*}}}(-1)^{\Lambda_{\gamma^{*}}}\left(\varepsilon_{\mu^{\gamma^{*}}}^{\Lambda_{\gamma^{*}}}\right)^{*} \varepsilon_{\nu}^{\Lambda_{\gamma^{*}}}
$$

Inserting the expansion in Eq. (6), we obtain the following invariant expression:

$$
T_{\mathrm{DVCS}}=\frac{e^{3}}{q^{2}}\left(j_{\mathrm{DVCS}}^{\mu} \varepsilon_{\mu}^{\Lambda_{\gamma^{*}} *}\right)\left(J_{\mathrm{DVCS}}^{\nu} \varepsilon_{\nu}^{\Lambda_{\gamma^{*}}}\right),
$$

where the photon polarization vector contracted with the hadron current is evaluated in the hadron scattering plane, and it is therefore rotated by a phase,

$$
\varepsilon_{\mu}^{\Lambda_{\gamma}^{*}} \text { (hadron) }=e^{-i \Lambda_{\gamma}^{*} \phi} \varepsilon_{\mu}^{\Lambda_{\gamma}^{*}} \text { (lepton). }
$$

This phase determines the $\phi$ dependence of the DVCS cross section. The $\mathrm{BH}$ cross section has only kinematic $\phi$ dependence through four-vector products of the type $(k \Delta),\left(k q^{\prime}\right), \ldots$, where $k$ lies in the lepton plane, and $\Delta, q^{\prime} \ldots$, lie in the hadron plane. The BH-DVCS interference term has both a phase dependence from the DVCS contribution and a kinematic dependence on the angle $\phi$ through the $\mathrm{BH}$ contribution. All of these contributions were calculated explicitly in Ref. [9] carefully separating out the phase dependence from the kinematic one. The phase dependence is not made explicit in the harmonic expansion formalism of BKM, and we surmise that its different (or lack of) treatment is at the origin of the numerical discrepancies shown in this paper.

We focus on the cross sections for either an unpolarized or a polarized electron scattering off an unpolarized nucleon which are, respectively, given by

$$
\begin{gathered}
\sigma_{U U}=\sigma_{U U}^{\mathrm{BH}}+\sigma_{U U}^{\mathrm{DVCS}}+\sigma_{U U}^{\mathcal{I}}, \\
\sigma_{L U}=\sigma_{L U}^{\mathrm{DVCS}}+\sigma_{L U}^{\mathcal{I}} .
\end{gathered}
$$

The detailed structures of the BH, DVCS, and BH-DVCS interference contributions to the cross sections in Eqs. (13) and (14) given in Ref. [9] in the Born approximation read

$$
\begin{gathered}
\sigma_{U U}^{\mathrm{BH}}=\Gamma\left|T_{\mathrm{BH}}\right|^{2}=\frac{\Gamma}{t^{2}}\left[A_{\mathrm{BH}}\left(F_{1}^{2}+\tau F_{2}^{2}\right)+B_{\mathrm{BH}} \tau G_{M}^{2}\right], \\
\sigma_{U U}^{\mathrm{DVCS}}=\frac{\Gamma}{Q^{2}(1-\epsilon)}\left\{F_{U U, T}+\epsilon F_{U U, L}+\epsilon \cos 2 \phi F_{U U}^{\cos 2 \phi}+\sqrt{\epsilon(\epsilon+1)} \cos \phi F_{U U}^{\cos \phi}\right\}, \\
\sigma_{L U}^{\mathrm{DVCS}}=\frac{\Gamma}{Q^{2}(1-\epsilon)}(2 h) \sqrt{2 \epsilon(1-\epsilon)} \sin \phi F_{L U}^{\sin \phi}, \\
\sigma_{U U}^{\mathcal{I}}=e_{l} \frac{\Gamma}{Q^{2}|t|}\left\{A_{U U}^{\mathcal{I}} \Re e\left(F_{1} \mathcal{H}+\tau F_{2} \mathcal{E}\right)+B_{U U}^{\mathcal{I}} G_{M} \Re e(\mathcal{H}+\mathcal{E})+C_{U U}^{\mathcal{I}} G_{M} \Re e \widetilde{H}+\frac{\sqrt{t_{0}-t}}{Q} F_{U U}^{\mathcal{I}, t w 3}\right\}, \\
\sigma_{L U}^{\mathcal{I}}=e_{l} \frac{\Gamma}{Q^{2}|t|}\left\{A_{L U}^{\mathcal{I}} \Im m\left(F_{1} \mathcal{H}+\tau F_{2} \mathcal{E}\right)+B_{L U}^{\mathcal{I}} G_{M} \Im m(\mathcal{H}+\mathcal{E})+C_{L U}^{\mathcal{I}} G_{M} \Im m \widetilde{H},+\frac{\sqrt{t_{0}-t}}{Q} F_{L U}^{\mathcal{I}, t w 3}\right\},
\end{gathered}
$$


where $e_{l}$ is the lepton charge, $y=(q p) /(k p)$, $\tau=-t / 4 M^{2}, t_{0}$ is the minimum $t$ value allowed by taking the transverse four-momenta transfer $\Delta_{T} \geq 0$, and $\epsilon$, the ratio of longitudinal to transverse virtual photon flux in DVCS, is given by

$$
\epsilon \equiv \frac{1-y-\frac{1}{4} y^{2} \gamma^{2}}{1-y+\frac{1}{2} y^{2}+\frac{1}{4} y^{2} \gamma^{2}} .
$$

$F_{1}$ and $F_{2}$ are the Dirac and Pauli form factors; $A_{\mathrm{BH}}$ and $B_{\mathrm{BH}}$ are kinematic coefficients expressed in terms of fourvector products involving all the relevant four-momenta: the initial and final electron momenta, $k$ and $k^{\prime}$, the final photon momentum, $q^{\prime}, \Delta$, and the average proton momentum $P=\left(p+p^{\prime}\right) / 2$. In Eq. (15), we wrote their kinematic dependence on the relevant kinematic variables fore the process, $y, x_{B j}, t, Q^{2}$, and $\phi$. Their detailed expressions are given in Ref. [9]. The CFFs are defined, in the QCD factorization framework, as convolutions of the GPDs for each quark flavor, $q$, with the Wilson coefficients functions. At leading order, we have for, $\mathcal{F}_{q}=\left(\mathcal{H}_{q}, \mathcal{E}_{q}\right)$ and $\widetilde{\mathcal{F}}_{q}=\left(\widetilde{\mathcal{H}}_{q} \widetilde{\mathcal{E}}_{q}\right)$, respectively,

$\mathcal{F}_{q}(\xi, t)=\mathcal{C}\left(C^{+} F_{q}\right) \equiv \int_{-1}^{1} d x C^{+}(x, \xi) F_{q}(x, \xi, t)$,

$\widetilde{\mathcal{F}}_{q}(\xi, t)=\mathcal{C}\left(C^{-} \widetilde{F}_{q}\right) \equiv \int_{-1}^{1} d x C^{-}(x, \xi) \widetilde{F}_{q}(x, \xi, t)$,

with the leading order coefficients functions given by

$$
C^{ \pm}(x, \xi)=\frac{1}{x-\xi-i \epsilon} \mp \frac{1}{x+\xi-i \epsilon} .
$$

The GPDs observe crossing symmetry relations with respect to $x \rightarrow-x$, which allow us to introduce valence (symmetric) and quark singlet (antisymmetric) distributions (for a detailed discussion see Refs. [32,33]). In DVCS, the proton GPD is written in terms of the quark GPDs as

$$
H=\sum_{q} e_{q}^{2} H_{q},
$$

with $e_{q}$ being the quark charge. The neutron GPD can be obtained using isospin symmetry.

Although the full structure of the cross section was already given in Ref. [9], to facilitate data analyses and interpretations, we make the following observations:

(i) The $\mathrm{BH}$ cross section is cast in a form similar to $e p$ elastic scattering. However, due to the additional photon radiated from the electron in either the initial or final state, the virtual photon exchanged with the target is aligned along $\Delta$ at an angle $\phi$, and the kinematic coefficients, $A_{\mathrm{BH}}$ and $B_{\mathrm{BH}}$, multiplying the form factors, acquire a complicated dependence in $\phi$ [9]. A more physical interpretation of these terms can also be obtained by writing the coefficients combination,

$$
\epsilon_{\mathrm{BH}}=\left(1+\frac{B_{\mathrm{BH}}}{A_{\mathrm{BH}}}(1+\tau)\right)^{-1},
$$

measuring the exchanged virtual photon's longitudinal polarization relative to the transverse. Notice that $\epsilon_{\mathrm{BH}} \neq \epsilon$.

(ii) The DVCS structure functions are bilinear functions of the CFFs multiplied by kinematic coefficients that are directly related to the helicity structure for the process. We introduced a similar notation as in Refs. [13,26] defining $F_{U U, T}, F_{U U, L}, F_{U U}^{\cos \phi}, F_{U U}^{\cos 2 \phi}$, and $F_{L U}^{\sin \phi}$, where the first and second subscripts define the polarization of the beam and target, respectively, and the third subscript defines the polarization of the virtual photon. The superscripts refer to the azimuthal angular dependence associated with each structure function. Notice the structure of the multiplicative factors in each structure function: $F_{U U, T}$ has no $\sqrt{t_{0}-t} / Q$ factor and is therefore the dominant term at high $Q^{2} . F_{U U}^{\cos \phi}, F_{U U}^{\sin \phi}$ contain one helicity flip factor $\propto \sqrt{t_{0}-t} / Q . F_{U U}^{\cos 2 \phi}$ is a leading twist contribution associated with a double helicity flip, and it is both proportional to $\left(t_{0}-t\right) / M^{2}$ and suppressed by a factor $\alpha_{S}$ from the gluon coupling. Finally, $F_{U U, L}$, containing only twist-three GPDs, is given by the product of two single-flip terms yielding a multiplicative factor of $\left(t_{0}-t\right) / Q^{2}$.

(iii) The BH-DVCS interference contribution is expressed in terms of linear combinations of products of CFFs elastic form factors, $F_{1}$ and $F_{2}$, with the coefficients, $A_{U U}^{\mathcal{I}}, B_{U U}^{\mathcal{I}}, C_{U U}^{\mathcal{I}}$, which are functions of $\left(Q^{2}, x_{B j}, t, y, \phi\right)$ [9]. Similar to the DVCS contribution, there is no obvious connection between the various beam/target polarization configurations and the GPDs contributing to the structure functions. On the other hand, similar to the BH term, one can single out the contributions,

$$
F_{1} \mathcal{H}+\tau F_{2} \mathcal{E}, \quad G_{M}(\mathcal{H}+\mathcal{E}), \quad G_{M} \tilde{\mathcal{H}},
$$

where the electric and magnetic properties of the cross section are clearly separated out, and in addition, one has the equivalent of an axial charge term. The latter appears similarly to the parity violating term in elastic scattering. It is, however, parity conserving in DVCS-BH interference because of the presence of the extra photon emitted at the proton vertex.

For completeness, we list the expressions for both the $\mathrm{BH}$ and BH-DVCS interference terms in Appendix B. 


\section{A. Comparison with previous formulations}

In exclusive unpolarized scattering processes from the proton, it is expected that the magnetic contribution will appear suppressed with respect to the electric one due to the phase dependence of the spin flip amplitude describing this term. Indeed, in Ref. [9], we found that this holds specifically for DVCS, where the magnetic contribution to $\sigma_{U U}^{\mathcal{I}}$ appears multiplied by a smaller coefficient, $B_{U U}^{\mathcal{I}}$, than the electric form factor. This contribution represents, however, the most interesting term of the unpolarized cross section; since it contains the combination of CFFs, $\mathcal{H}+\mathcal{E}$, it brings us closer to getting a quantitative hold of angular momentum [1]. A goal of this paper is to help galvanize the efforts to extract information on this important quantity. Notice, in fact, that, as shown in Sec. III, in our formalism, the coefficient of the magnetic term, $B_{U U}^{\mathcal{I}}$, is larger than the coefficient of the axial term, $C_{U U}^{\mathcal{I}}$, thus bringing this term within current experimental grasp. In the BKM formalism, on the contrary, the coefficient $C_{U U}^{\mathcal{I}}$ is larger than $B_{U U}^{\mathcal{I}}$.
The analytic forms of the coefficients $A_{U U}^{\mathcal{I}}, B_{U U}^{\mathcal{I}}$, and $C_{U U}^{\mathcal{I}}$, evaluated using the BKM formalism, are also given in Appendix C. Another difference is in the twist-three GPD contributions, written in detail in the next section, which we define along the lines of the GPD decomposition of the correlation function of Ref. [34]. This allows us for the first time to give a physical interpretation of the various twistthree contributions in terms of orbital angular momentum and spin orbit contributions.

The numerical evaluation of the differences with the BKM formalism presented in Refs. [14-16] is presented in Sec. III in various kinematic regimes.

\section{B. Twist three}

We discuss the structure of the BH-DVCS interference term at twist three, in view of the fact that it contains GPDs describing the longitudinal component of orbital angular momentum, $\widetilde{E}_{2 T}$, the spin orbit term $\left(2 \widetilde{\mathcal{H}}_{2 T}^{\prime}+\mathcal{E}_{2 T}^{\prime}\right)[35,36]$, and terms related to transverse angular momentum, $H_{2 T}$,

$$
\begin{aligned}
F_{U U}^{\mathcal{I}, t w 3}= & A_{U U}^{(3) \mathcal{I}}\left[F_{1}\left(\Re e\left(2 \widetilde{\mathcal{H}}_{2 T}+\mathcal{E}_{2 T}\right)-\Re e\left(2 \widetilde{\mathcal{H}}_{2 T}^{\prime}+\mathcal{E}_{2 T}^{\prime}\right)\right)+F_{2}\left(\Re e\left(\mathcal{H}_{2 T}+\tau \widetilde{\mathcal{H}}_{2 T}\right)-\Re e\left(\mathcal{H}_{2 T}^{\prime}+\tau \widetilde{\mathcal{H}}_{2 T}^{\prime}\right)\right)\right] \\
& +B_{U U}^{(3) \mathcal{I}} G_{M}\left(\Re e \widetilde{\mathcal{E}}_{2 T}-\Re e \widetilde{\mathcal{E}}_{2 T}^{\prime}\right) \\
& +C_{U U}^{(3) \mathcal{I}} G_{M}\left[2 \xi\left(\Re e \mathcal{H}_{2 T}-\Re e \mathcal{H}_{2 T}^{\prime}\right)-\tau\left(\Re e\left(\widetilde{\mathcal{E}}_{2 T}-\xi \mathcal{E}_{2 T}\right)-\Re e\left(\widetilde{\mathcal{E}}_{2 T}^{\prime}-\xi \mathcal{E}_{2 T}^{\prime}\right)\right)\right] .
\end{aligned}
$$

For a polarized electron beam, we obtain a structure analogous to the unpolarized case, where the $\Re e$ parts of the CFFs are replaced with the $\Im m$ parts, namely,

$$
\begin{aligned}
F_{L U}^{\mathcal{I}}= & A_{L U}^{(3) \mathcal{I}}\left[F_{1}\left(\Im m\left(2 \widetilde{\mathcal{H}}_{2 T}+\mathcal{E}_{2 T}\right)-\Im m\left(2 \widetilde{\mathcal{H}}_{2 T}^{\prime}+\mathcal{E}_{2 T}^{\prime}\right)\right)+F_{2}\left(\Im m\left(\mathcal{H}_{2 T}+\tau \widetilde{\mathcal{H}}_{2 T}\right)-\Im m\left(\mathcal{H}_{2 T}^{\prime}+\tau \widetilde{\mathcal{H}}_{2 T}^{\prime}\right)\right)\right] \\
& +B_{L U}^{(3) \mathcal{I}} G_{M}\left(\Im m \widetilde{\mathcal{E}}_{2 T}-\Im m \widetilde{\mathcal{E}}_{2 T}^{\prime}\right) \\
& +C_{L U}^{(3) \mathcal{I}} G_{M}\left[2 \xi\left(\Im m \mathcal{H}_{2 T}-\Im m \mathcal{H}_{2 T}^{\prime}\right)-\tau\left(\Im m\left(\widetilde{\mathcal{E}}_{2 T}-\xi \mathcal{E}_{2 T}\right)-\Im m\left(\widetilde{\mathcal{E}}_{2 T}^{\prime}-\xi \mathcal{E}_{2 T}^{\prime}\right)\right)\right] .
\end{aligned}
$$

The coefficients, $A_{U U}^{(3) \mathcal{I}}, B_{U U}^{(3) \mathcal{I}}, C_{U U}^{(3) \mathcal{I}}$, and $A_{L U}^{(3) \mathcal{I}}, B_{L U}^{(3) \mathcal{I}}, C_{L U}^{(3) \mathcal{I}}$, are written in terms of four-vector products involving all relevant variables, for the electron, $k, k^{\prime}$, final photon, $q^{\prime}$, momentum transfer, $\Delta$, and average proton momentum, $P$. Similarly to the twist-two case, they can be expressed in terms of the set of variables $\left(Q^{2}, x_{B j}, t, y, \phi\right)$. Their specific expressions are given for the first time in Ref. [9]. A comparison with BKM cannot be performed due to the inherently different structure of their dynamic twistthree expressions. Note for the twist-three CFFs, $\mathcal{H}_{2 T}, \mathcal{E}_{2 T} \ldots$, we use the same notation as for the twisttwo case, by defining them through the convolution with the leading order Wilson coefficient functions given in Eq. (21). The possible role of an explicit qgq term is beyond the scope of this paper.

The notation for the twist-three GPDs is illustrated in Table I where we show, along with our notation, their quark-proton polarization configuration, the corresponding
TABLE I. Twist-three GPDs and their helicity content. In the first column, we show the GPDs notation for this paper. The second column shows the quark and proton polarizations. The third column shows the analogous configurations in the TMD sector. Finally, the fourth column shows the corresponding notation from Ref. [34]. The asterisk denotes naive T-odd twistthree TMDs (we define $\tau=\frac{t_{o}-t}{4 M^{2}}$ ).

\begin{tabular}{lccc}
\hline \hline GPD & $P_{q} P_{p}$ & TMD & Ref. [34] \\
\hline$H^{\perp}$ & UU & $f^{\perp}$ & $2 \widetilde{H}_{2 T}+E_{2 T}$ \\
$\widetilde{H}_{L}^{\perp}$ & LL & $g_{L}^{\perp}$ & $2 \widetilde{H}_{2 T}^{\prime}+E_{2 T}^{\prime}$ \\
$H_{L}^{\perp}$ & UL & $f_{L}^{\perp(*)}$ & $\widetilde{E}_{2 T}-\xi E_{2 T}$ \\
$\widetilde{H}^{\perp}$ & LU & $g^{\perp(*)}$ & $\widetilde{E}_{2 T}^{\prime}-\xi E_{2 T}^{\prime}$ \\
$E^{\perp}$ & $\mathrm{UT}$ & $f_{T}^{(*)}$ & $H_{2 T}+\tau \widetilde{H}_{2 T}$ \\
$\widetilde{E}^{\perp}$ & $\mathrm{LT}$ & $g_{T}^{\prime}$ & $H_{2 T}^{\prime}+\tau \widetilde{H}_{2 T}^{\prime}$ \\
\hline \hline
\end{tabular}


notation in the TMD sector, and the notation from Ref. [34] (see also Table I in Ref. [9]). The notation follows the one adopted for TMDs [37,38], namely,

(i) $H$ and $f$ correspond to the vector coupling in the parametrization of the quark-proton correlation function.

(ii) $\widetilde{H}$ and $g$ correspond to axial-vector coupling.

(iii) The $\perp$ superscript indicates an unsaturated transverse momentum index in the correlation function's coefficient [35].

(iv) The subscript $L(T)$ involves the amplitude for a longitudinally (transversely) polarized target.

\section{Azimuthal angular dependence}

The azimuthal angular, $\phi$, dependence is a key feature of the cross section, appearing with different capacities in the description of the BH, DVCS, and BH-DVCS interference contributions. In electron scattering exclusive reactions, the cross section assumes a characteristic dependence on the phase, $\phi$, which originates from rotating the virtual photon polarization vector, $\varepsilon_{\mu}^{\Lambda_{\nu}^{*}}$, from the leptonic to the hadronic plane (see, e.g., Refs. [31,39] and the detailed reiteration for deeply virtual scattering in Refs. [12,13]). This dependence allows us, in general, to single out the contributions from the overlap of different transverse and longitudinal amplitudes (e.g., $\sigma_{T}, \sigma_{T T}, \sigma_{L T} \ldots$ ) describing different physics content.

In the specific case of exclusive photoproduction, we distinguish between the $\mathrm{BH}$ and DVCS processes. In $\mathrm{BH}$, there are only two structure functions, i.e., the proton elastic form factors; therefore, similar to elastic scattering, we do not organize the cross section by writing out the various virtual photon polarization components. The dependence on $\phi$ is purely kinematic.

For the DVCS and BH-DVCS contributions, we set the virtual photon for DVCS, $q$, along the $z$ axis in the laboratory frame, while the virtual photon, $\Delta$, for the $\mathrm{BH}$ process is along $\Delta$, at an angle $\phi$ with respect to $q$.

As shown below, this mismatch results in a much more complicated dependence of the polarization-vector products contributing to the cross section, generating substantial $t$ and target mass corrections [9].

\section{Phase dependence of pure DVCS contribution}

The polarization vectors for the virtual photon of momentum $q$ along the negative $z$ axis in the laboratory frame are defined as

$$
\begin{gathered}
\varepsilon^{\Lambda_{\gamma^{*}}= \pm 1} \equiv \frac{1}{\sqrt{2}}(0 ; \mp 1, i, 0), \\
\varepsilon^{\Lambda_{\gamma^{*}}=0} \equiv \frac{1}{Q}\left(|\vec{q}| ; 0,0, q_{0}\right)=\frac{1}{\gamma}\left(\sqrt{1+\gamma^{2}} ; 0,0,1\right) .
\end{gathered}
$$

Notice that the DVCS helicity amplitudes are evaluated in the center of mass (c.m.) frame of the final photon-hadron system, which defines the hadron plane at an angle $\phi$ with respect to the lepton plane [9]. The cross section is evaluated by transforming to the lepton plane rotating the polarization vectors defining the helicty amplitude, $f_{\Lambda \Lambda^{\prime}}^{\Lambda_{\gamma}^{*} \Lambda_{\gamma}^{\prime}}$, by $-\phi$ about the $z$ axis. Another way to express this is that the lepton produces a definite helicity virtual photon which we take along the $z$ axis in the lepton plane; however, the virtual photon's interaction with the target occurs in the hadron plane which is rotated through an azimuthal angle $\phi$. The phase dependence of the DVCS contribution to the cross section is a consequence of such a rotation about the axis where the virtual photon lies [30,31]. The $\phi$ rotation about the $z$ axis changes the phase of the transverse components and leaves the longitudinal polarization vector unchanged as

$$
\varepsilon^{\Lambda_{\gamma^{*}}= \pm 1} \rightarrow \frac{e^{-i \Lambda_{\gamma^{*} \phi}}}{\sqrt{2}}(0, \mp 1, i, 0) .
$$

The ejected (real) photon polarization vectors read

$$
\begin{aligned}
\varepsilon^{\Lambda_{y}^{\prime}= \pm 1} \equiv & \frac{1}{\sqrt{2}}(0 ; \mp \cos \theta \cos \phi+i \sin \phi \\
& \mp \cos \theta \sin \phi+i \cos \phi, \pm \sin \theta)
\end{aligned}
$$

$\varepsilon^{\Lambda_{y}^{\prime}}$, in principle, also undergoes a phase rotation; however, this phase rotation does not contribute to the cross section due to the completeness relation obtained summing over the physical (on-shell) states [40],

$$
\sum_{\Lambda_{\gamma}^{\prime}}\left(\varepsilon_{\mu}^{\Lambda_{\gamma}^{\prime}}\left(q^{\prime}\right)\right)^{*} \varepsilon_{\nu}^{\Lambda_{\nu}^{\prime}}\left(q^{\prime}\right)=-g_{\mu \nu} .
$$

\section{Phase dependence of BH-DVCS interference term}

The same treatment described above is applied to the BH-DVCS interference term. Here, the different polarizations allow us to distinguish the twist-two and twistthree terms as

$$
\begin{gathered}
\text { twist } 2 \rightarrow \sum_{\Lambda_{\nu}^{*}= \pm 1}\left(\varepsilon_{\mu}^{\Lambda_{\gamma}^{*}}\right)^{*} \varepsilon_{\nu}^{\Lambda_{\gamma}^{*}}=\cos \phi g_{\mu \nu}^{T}-\sin \phi \varepsilon_{\mu \nu}^{T}, \\
\text { twist } 3 \rightarrow\left(\varepsilon_{\mu}^{\Lambda_{\gamma}^{*}=0}\right)^{*} \varepsilon_{\nu}^{\Lambda_{\nu}^{*}=0}=g_{\mu \nu}^{L},
\end{gathered}
$$

where the components of $g_{\mu \nu}^{L}$ are $g_{00}^{L}=1+\nu^{2} / Q^{2}$, $g_{03}^{L}=g_{30}^{L}=\nu \sqrt{\nu^{2}+Q^{2}} / Q^{2}$, and $g_{33}^{L}=\nu^{2} / Q^{2}$.

In addition to the phase dependence, differently from the pure DVCS term where the azimuthal angular dependence resides entirely in the phase factors, the BH-DVCS contribution contains a $\phi$ dependence of kinematic origin. The kinematic $\phi$ dependence arises from the orientation of the $\Delta$ vector which lies at an angle $\phi$ in the hadronic plane and 
generates a $\phi$ dependence through factors of four-vector products $(k \Delta)$ in the coefficients of the structure functions similar to the $\mathrm{BH}$ case. As a result, we single out an overall multiplicative $\cos \phi$ term in the twist-two contribution to $\sigma_{U U}^{\mathcal{I}}$ and a $\sin \phi$ term in the twist-two contribution to $\sigma_{L U}^{\mathcal{I}}$, which originate from the phase dependence arising from the helicity amplitudes of the hadronic current. The coefficients contain also the kinematic $\phi$ dependence as explained above. A similar situation is found at twist three where the phase dependence cancels out since for the virtual photon $e^{-i \Lambda_{\gamma^{*}} \phi}$ is 1 as a consequence of its longitudinal polarization; thus, the $\phi$ dependence is entirely of kinematic origin.

This result seems at variance with the representation given in the original harmonics expansion of approach Refs. $[15,16]$. However, one should notice that in these papers the distinction between the $\phi$ dependence from the phase of the polarization vectors and the $\phi$ dependence from the kinematics is not evident. This point of departure of the two formalisms is important as also addressed in Refs. [23-25], where the question of $t$ and target mass corrections resulting from the different choices of the orientation of $\Delta$ has been also studied. In particular, it is important to establish a pathway in the decomposition of subleading twist terms which in our case clearly result from the $\phi$ dependence from the "phase" of the hadronic current. This ensures that what enters into our twist-three cross section terms are directly due to twist-three GPDs and not to kinematically suppressed terms. These issues seem to have prevented so far a clean extraction of information from data [41].

\section{NUMERICAL RESULTS}

In this section, we present numerical evaluations of the $\mathrm{BH}, \mathrm{DVCS}$, and BH-DVCS interference terms evaluated in Ref. [9], emphasizing the feature of our new formalism as compared to previous approaches discussed in Sec. II.

We compare results obtained both in the helicity amplitudes and in the BKM formulations [15,16], for various observables entering the unpolarized cross section at kinematic settings ranging from recent measurements at Jefferson Lab [42,43] to a $24 \mathrm{GeV}$ fixed target scenario [27] and the EIC $[28,29]$. Since the goal of this paper is to highlight the new features of the framework for exclusive electoproduction as compared to BKM, we restrain from discussing issues involving fits to the cross section, the extraction of the CFFs from experimental data, and the modeling of GPDs based on DVCS data. These topics will be discussed in upcoming publications.

We start from showing in Fig. 1 what is perhaps the biggest consequence of our new framework for deeply virtual exclusive scattering on an unpolarized proton: the dependence on the scale, $Q^{2}$, of the dominant contribution to the BH-DVCS interference matrix element, $\Re e\left(F_{1} \mathcal{H}+\tau F_{2} \mathcal{E}\right)$. The extraction of this quantity in our formalism is consistent with a slow $Q^{2}$ dependence as

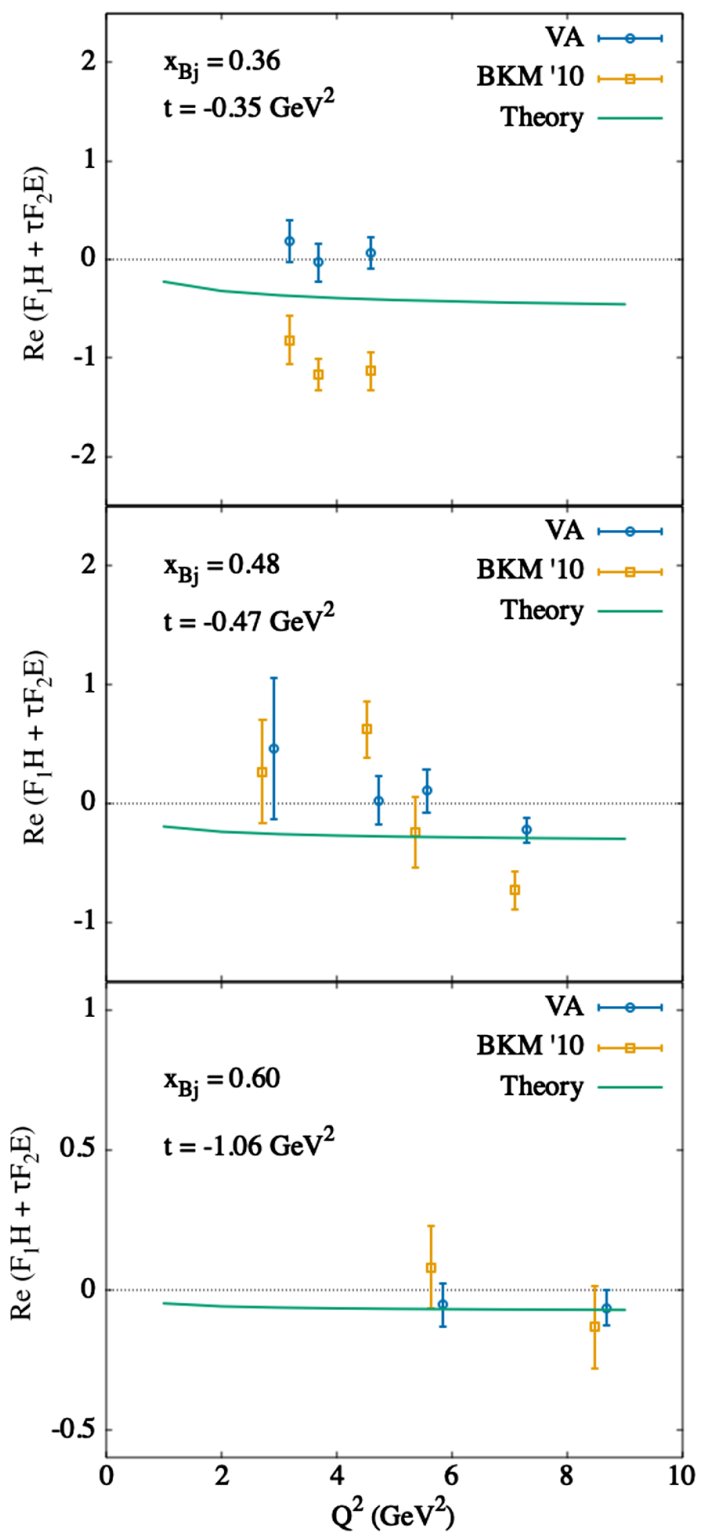

FIG. 1. The CFFs combination, $F_{1}(t) \Re e \mathcal{H}+\tau F_{2}(t) \Re e \mathcal{E}$, corresponding to the first term in Eq. (18), plotted vs $Q^{2}$. This quantity was extracted from data [42] using the formalism presented in this paper and in Ref. [9] (blue circles) and using the formalism of Ref. [16] (orange squares). The curves in the figure illustrate the effect of perturbative QCD evolution, calculated using the GPD parametrization in Refs. [45,46].

predicted by the perturbative QCD evolution equations for GPDs $[5,32,44]$. In the BKM formalism, on the contrary, oscillations appear which could indicate spurious $Q^{2}$ dependence resulting from the approximations taken in the cross section coefficients.

Notice that, although BKM use the harmonics based formulation where, in particular, the $\sigma_{U U}^{\mathcal{I}}$ cross section is not organized in terms of $A_{U U}^{\mathcal{I}}, B_{U U}^{\mathcal{I}}, C_{U U}^{\mathcal{I}}$, one can retrieve equivalent expressions by rearranging the various harmonics contributions. These expressions are displayed in 

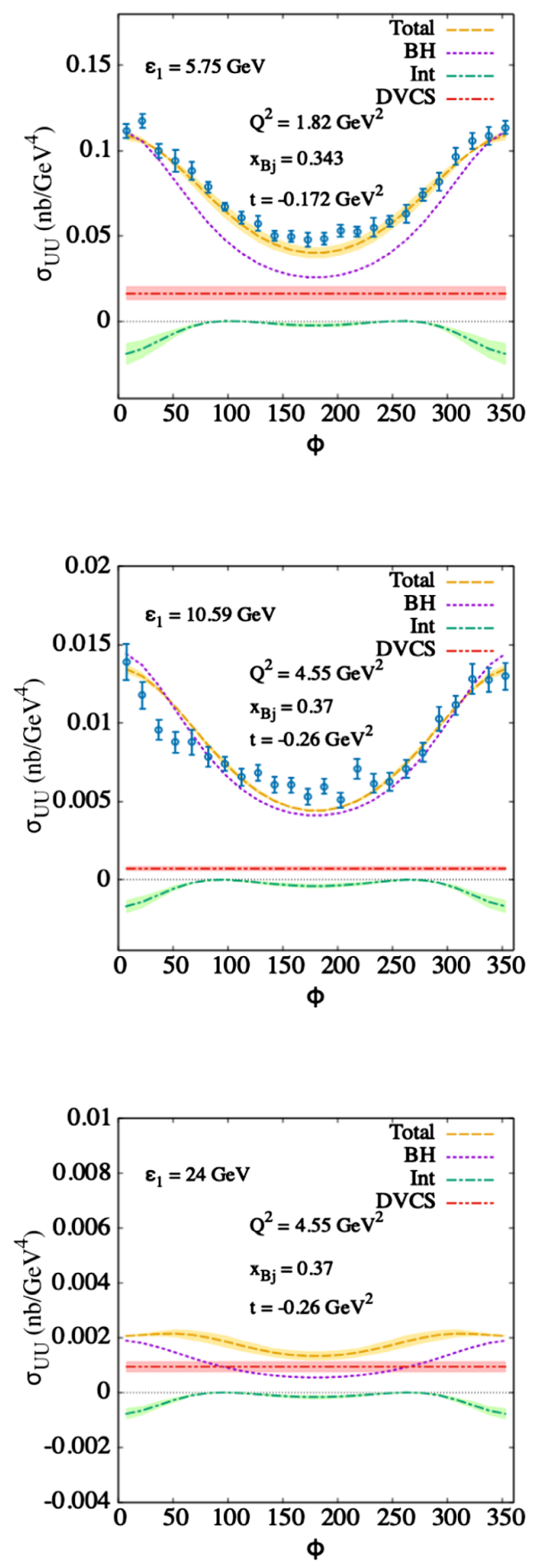

FIG. 2. The cross section $\sigma_{U U}$, Eq. (13) for the kinematic bins from Ref. [43] with initial electron energy $\epsilon_{1}=5.75 \mathrm{GeV}$, $Q^{2}=1.8 \mathrm{GeV}^{2}, \quad t=-0.172 \mathrm{GeV}^{2}, x_{B j}=0.34$ (top panel) [42]; $\epsilon_{1}=11.5 \mathrm{GeV}, Q^{2}=4.5 \mathrm{GeV}^{2}, t=-0.29 \mathrm{GeV}^{2}, x_{B j}=$ 0.37 (second panel); and for projected values of a fixed target experiment at $\epsilon_{1}=24 \mathrm{GeV}$ (third panel). The curves correspond to the contributions from $\sigma_{U U}$ Eq. (13), $\sigma_{U U}^{\mathrm{BH}},(15), \sigma_{U U}^{\mathrm{DVCS}},(16)$, and $\sigma_{U U}^{\mathcal{I}}$, (18), calculated in the laboratory system.
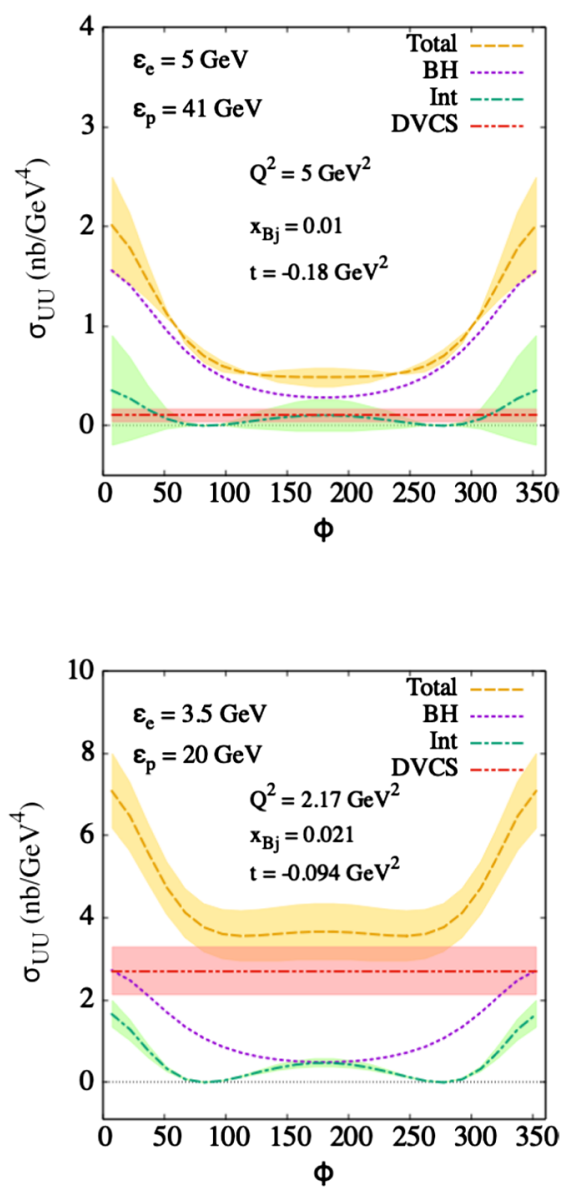

FIG. 3. The cross section $\sigma_{U U}$ in a collider setting kinematics for an EIC with initial electron energy $\epsilon_{e}=5 \mathrm{GeV}$, initial proton energy $\epsilon_{p}=41 \mathrm{GeV}$, and kinematic bin $x_{B j}=0.01$, $t=-0.18 \mathrm{GeV}^{2}$, and $Q^{2}=5 \mathrm{GeV}^{2}$ of the US-based EIC [29] (top), and initial electron energy $\epsilon_{e}=3.5 \mathrm{GeV}$, initial proton energy $\epsilon_{p}=20 \mathrm{GeV}$, and kinematic bin $x_{B j}=0.021$, $t=-0.094 \mathrm{GeV}^{2}$, and $Q^{2}=2.17 \mathrm{GeV}^{2}$ of the China-based EIC [28] (bottom).

Appendix C. We note that even by doing so, the analytic comparison between the two formulations represents a formidable task due to the inherent complications arising from the different choices of variables for the lengthy coefficients.

(i) In Fig. 2, we present the cross section $\sigma_{U U}$, Eq. (13), with the separate contributions, $\sigma_{U U}^{\mathrm{BH}},(15), \sigma_{U U}^{\mathrm{DVCS}}$, (16), and $\sigma_{U U}^{\mathcal{I}},(18)$, calculated in the Virginia (VA) framework. We consider three different settings in the laboratory system with electron beam energies: $\left(k_{e}\right)_{o}=\epsilon_{1}=5.75,11.5,24 \mathrm{GeV}$, and correspondingly increasing $Q^{2}$ values. The experimental data 

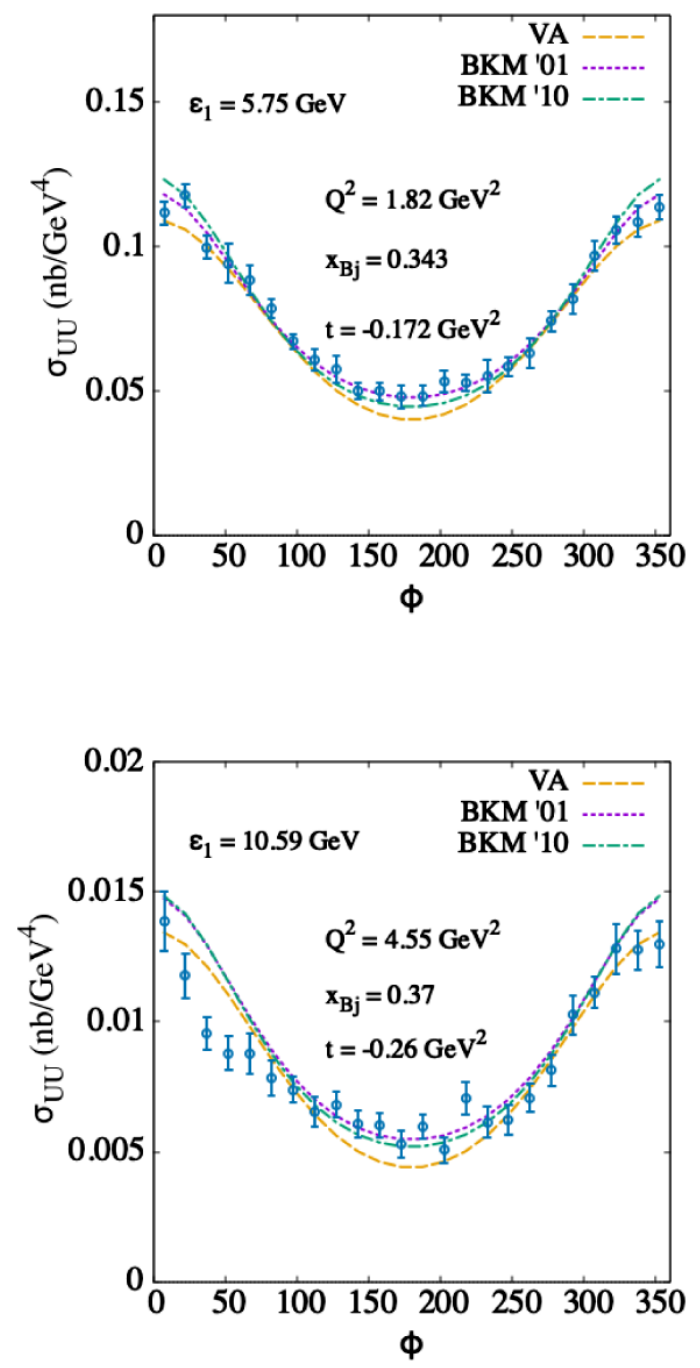

FIG. 4. Total unpolarized cross section with VA theory CFFs: (top) $\quad \epsilon_{1}=5.7 \mathrm{GeV}, \quad Q^{2}=1.8 \mathrm{GeV}^{2}, \quad x_{B j}=0.34$, $t=-0.17 \mathrm{GeV}^{2}$; (bottom) $\epsilon_{1}=10.6 \mathrm{GeV} \quad Q^{2}=4.5 \mathrm{GeV}^{2}$, $x_{B j}=0.37, t=-0.26 \mathrm{GeV}^{2}$.

are from Ref. [43] (top panel) and Ref. [42] (middle panel). The $24 \mathrm{GeV}$ setting [27] is becoming an exciting possibility that will allow further explorations of GPDs and the 3D structure of the nucleon in a wide kinematic range. In particular, the access to larger $Q^{2}$ values in the valence region will allow us to settle many issues related to power corrections and the onset of QCD factorization. The theoretical predictions for DVCS and the DVCS-BH interference were calculated at leading twist using GPDs from the spectator model in [45-47], summarized in Sec. III A. We underline that these are predictions, not fits, where the model parameters were fixed using constraints from experiments other than DVCS. The latter include a recent nucleon form factor and parton distribution function (PDF) measurements. These curves show a realistic picture of the relative sizes of the various contributions. The $\mathrm{BH}$ term is known to high precision, since its calculation is based on QED, the only unknowns being the nucleon form factors at low four-momenta transfer, $t$, where their uncertainty is small. The uncertainty band in the figure refers to the error from the fit in $[45,47]$.

(ii) The various contributions to the unpolarized cross section are shown in Fig. 3 for collider configurations at the EIC typical kinematic setting (upper panel) [29] and at EIcC kinematics (lower panel) [28].

(iii) In Fig. 4, we compare three cross section formulations: the present framework (VA), the formulations of Ref. [15] (BKM'01), and Ref. [16] (BKM'10), respectively. It should be noticed that the quantity plotted, $\sigma_{U U}$, is the sum of $\mathrm{BH}$, DVCS, and BH-DVCS interference contributions. All three calculations use the same $\mathrm{BH}$ cross section. The DVCS and DVCS-BH interference terms use the same CFFs values displayed in Table II, but they differ in the analytic form of the coefficients from the three different formulations. One can see sensible discrepancies between the VA and BKM calculations. It should be noticed that the differences are suppressed; i.e., they

TABLE II. Value of Compton form factors using Virginia Reggeized spectator model and global extraction from KM 15 [48] and KM 10a [49].

\begin{tabular}{lccccccccccc}
\hline \hline CFF & $x_{B j}$ & $-t\left(\mathrm{GeV}^{2}\right)$ & $Q^{2}\left(\mathrm{GeV}^{2}\right)$ & $\operatorname{Re} H$ & $\operatorname{Re} E$ & $\operatorname{Re} \widetilde{H}$ & $\operatorname{Re} \widetilde{E}$ & $\operatorname{Im} H$ & $\operatorname{Im} E$ & $\operatorname{Im} \widetilde{H}$ & $\operatorname{Im} \widetilde{E}$ \\
\hline VA & 0.34 & 0.17 & 1.82 & -0.897 & -0.541 & 0.244 & 2.207 & 4.842 & 1.806 & 1.131 & 5.383 \\
VA & 0.37 & 0.26 & 4.55 & -0.884 & -0.424 & 0.312 & 2.900 & 3.702 & 1.298 & 0.911 & 3.915 \\
KM15 & 0.34 & 0.17 & 1.82 & -2.254 & 2.212 & 1.399 & 141.362 & 3.506 & $\cdots$ & 1.565 & $\cdots$ \\
KM15 & 0.37 & 0.26 & 4.55 & -2.143 & 1.990 & 1.098 & 87.385 & 2.793 & $\cdots$ & 1.371 & $\ldots$ \\
KM10a & 0.34 & 0.17 & 1.82 & -1.513 & 1.583 & $\cdots$ & 40.863 & 3.783 & $\cdots$ & $\cdots$ & $\ldots$ \\
KM10a & 0.37 & 0.26 & 4.55 & -1.574 & 1.518 & $\cdots$ & 22.146 & 3.147 & $\cdots$ & $\cdots$ & $\cdots$ \\
\hline \hline
\end{tabular}



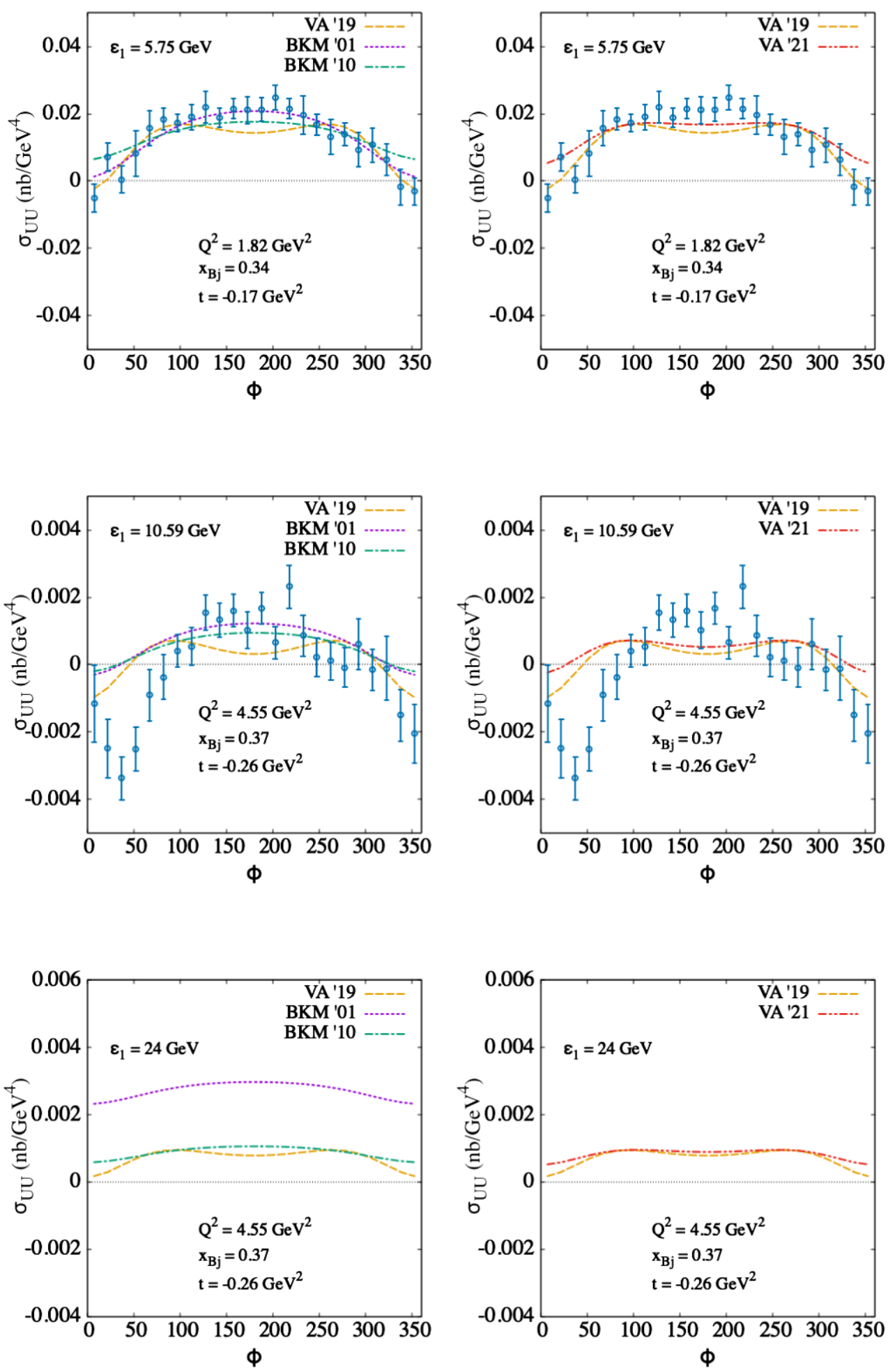

FIG. 5. Comparison of the interference plus DVCS contributions to the cross section, $\sigma_{U U}^{\mathcal{I}}+\sigma_{U U}^{\mathrm{DVCS}}$, calculated using the frameworks from the VA and BKM groups, respectively. Left panels: same notation and kinematic bins as in Fig. 2. Right panels: comparison between the formalism of Ref. [9], VA'19, the same formulation including the higher order gauge fixing coefficients given in Appendix C, VA'21, and BKM'10. 


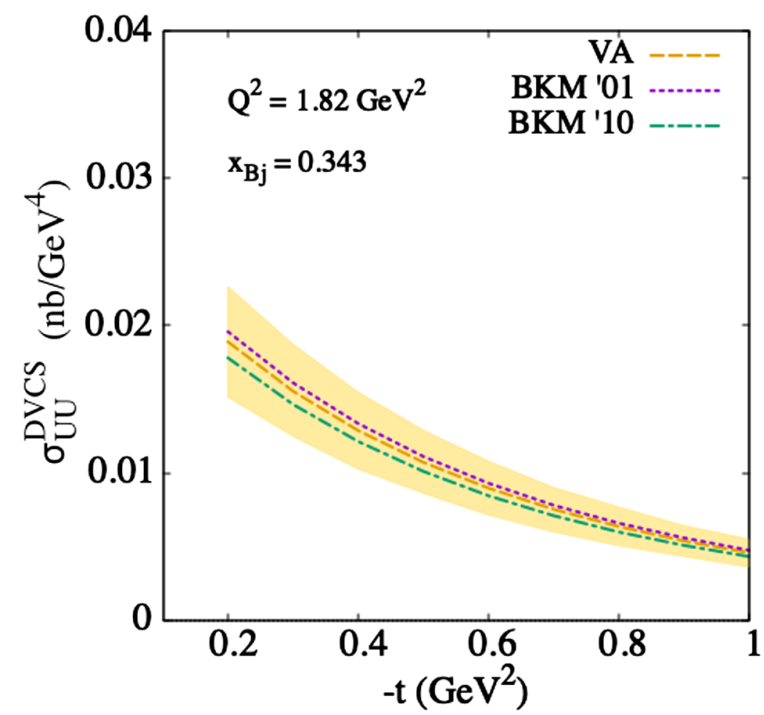

FIG. 6. Comparison of the unpolarized cross section, $\sigma_{U U}^{\mathrm{DVCS}}$, Eq. (16), obtained in the VA and BKM frameworks, respectively. $\sigma_{U U}^{\mathrm{DVCS}}$ is plotted vs $-t$ using the CFFs from the Reggeized diquark model. The error band represents a theoretical error from the Reggeized diquark model fit.

appear smaller in value owing to the fact that the cross section is dominated by the $\mathrm{BH}$ contribution which is the same in both the VA and BKM formulations. The differences will, however, affect the extraction of the CFFs.

(iv) To better describe the size of the difference between the two formalisms, we compare the $\sigma_{U U}^{\mathcal{I}}+$ $\sigma_{U} U^{\mathrm{DVCS}}$ terms in Fig. 5. One can see clear discrepancies between the two frameworks that do not seem to decrease with increasing electron energy. Notice that the VA formalism has different features of the $\phi$ modulations characterizing the cross section at central values of $\phi$.

This feature shows up clearly in the lhs panels. On the rhs, the effect of the extra terms originating from gauge invariance preserving coefficients is displayed. The impact of these terms tends to disappear with larger $Q^{2}$. It should be stressed that none of the curves shown in the figure correspond to a fit of the DVCS data in that the values of the CFFs are theoretical predictions.

(i) The $t$ dependence of the DVCS contribution, $\sigma_{U U}^{\mathrm{DVCS}}$, is presented in Fig. 6, for the kinematic bin, $\epsilon_{1}=5.75 \mathrm{GeV}, Q^{2}=1.8 \mathrm{GeV}^{2}, x_{B j}=0.34$ (other kinematics display a similar trend). One can see that for this term the improved calculation of Ref. [16] brings the VA and BKM evaluations closer.

(ii) To interpret the origin of the BKM-VA discrepancies, in Figs. 7 and 8 we juxtapose calculations using the VA formalism (left panels) to the BKM formalism (right panels). Figure 7 shows the same quantity, $\sigma_{U U}^{\mathrm{DVCS}}+\sigma_{U U}^{\mathcal{I}}$, as in Fig. 5 displaying the contributions to the latter from the three terms, $A_{U U}^{\mathcal{I}}\left(F_{1} \mathcal{H}+\tau F_{2} \mathcal{E}\right), B_{U U}^{\mathcal{I}} \propto G_{M}(\mathcal{H}+\mathcal{E})$, and $C_{U U}^{\mathcal{I}} \propto G_{M} \widetilde{H}$ (Eq. (18). From the figure, one can see that the term proportional to $A_{U U}^{\mathcal{I}}$ dominates the cross section. What is striking is the different weights that the $A_{U U}^{\mathcal{I}}, B_{U U}^{\mathcal{I}}$, and $C_{U U}^{\mathcal{I}}$ terms carry, respectively, in the VA and BKM frameworks. The differences with the VA formalism are particularly striking for the axial term, $C_{U U}^{\mathcal{I}}$, which is both smaller in size and has a complex $\phi$ modulations for the VA case. These differences persist in the kinematic range of Jefferson Lab@12 GeV Ref. [42] (not shown in the figure). While the $A_{U U}^{\mathcal{I}}$ term dominates the VA cross section, the contribution from $C_{U U}^{\mathcal{I}}$ is important in the BKM case, especially with increasing energy. The uncertainty bands in the figure represent the theoretical error evaluated using the model in Ref. [45].

(iii) In Fig. 9, we compare in detail the coefficients, $A_{U U}^{\mathcal{I}}, B_{U U}^{\mathcal{I}}$, and $C_{U U}^{\mathcal{I}}$ for the BKM and VA formulations. In order to understand whether the discrepancies are due to terms proportional to $M^{2} / Q^{2}, t / Q^{2}$, we studied the behavior of the 

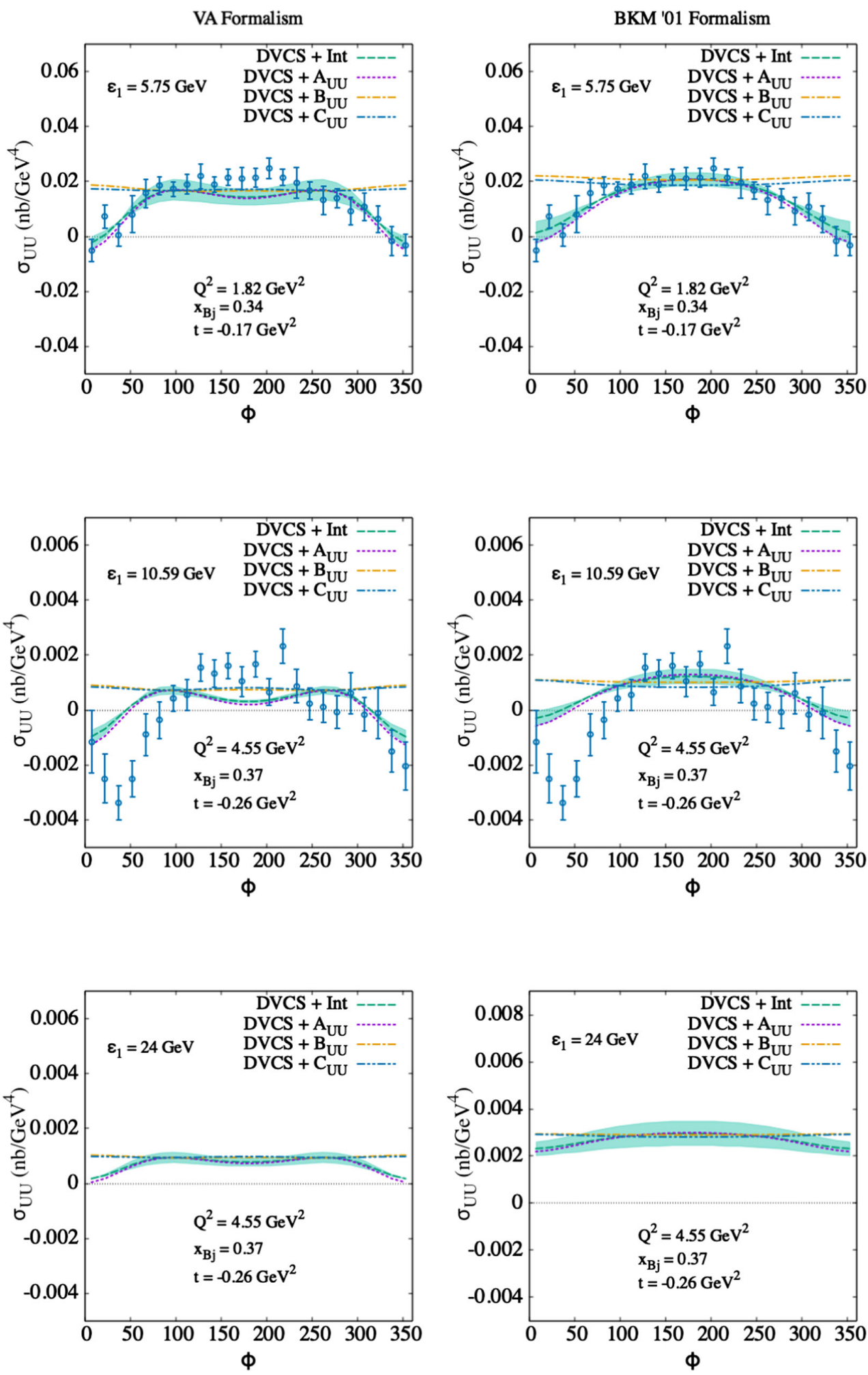

FIG. 7. BH-DVCS interference contribution to the cross section $\sigma_{U U}^{\mathcal{I}}$, Eq. (18) in the VA (left) and BKM (right) formalism. The initial electron energy is $\epsilon_{1}=5.75 \mathrm{GeV}$ from Ref. [43] (top panels), $\epsilon_{1}=10.5 \mathrm{GeV}$ Ref. [42] (middle panels), and for a projected value of a fixed target experiment at $\epsilon_{1}=24 \mathrm{GeV}$ (bottom panels). The curves correspond to the calculation at twist two using the Reggeized diquark model [45] for the electric current which appears in the cross section multiplied by $A_{U U}$, the magnetic term with coefficient $B_{U U}$, and the axial term, $C_{U U}$. 

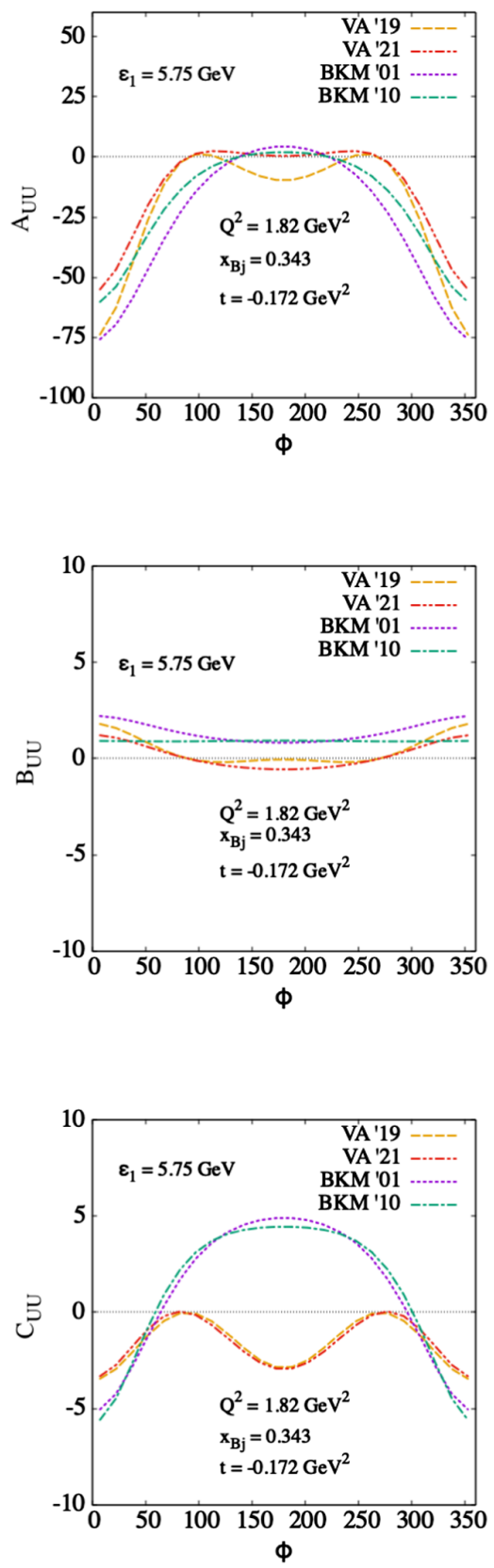

FIG. 8. Comparison of the kinematic coefficients, $A_{U U}$ (top), $B_{U U}$ (middle), and $C_{U U}$ (bottom) at the kinematic point $Q^{2}=1.82 \mathrm{GeV}^{2}, x_{B j}=0.34, t=-0.17 \mathrm{GeV}^{2}, \epsilon_{1}=5.75 \mathrm{GeV}$. coefficients vs $t$ and $Q^{2}$. The results shown in Fig. 9 represent the percentage deviations of the BKM'01 and BKM'10 coefficients from the VA ones, $A_{U U}$ (top), $B_{U U}$ (middle), and $C_{U U}$ (bottom), evaluated at $\phi=0$ (left panel) and $\phi=180$ degrees (right panel). On the lhs, we plot the percentage deviations as a function of $-t$, at $Q^{2}=1.8 \mathrm{GeV}^{2}$; on the rhs, they are plotted vs $Q^{2}$ at $-t=0.17 \mathrm{GeV}^{2}$. Notice that the differences among the approaches tend to subside at small $t$ and in the large $Q^{2}$ limit. Our findings substantiate the hypothesis that the treatment of $t$-dependent and target mass corrections is important, although no systematic effect can be singled out.

(iv) We also evaluated the impact of the coefficients of the twist-three contributions These are presented in Fig. 10. Our estimate shows that twistthree terms are small, of the same size of the $B_{U U}^{\mathcal{I}}, B_{U U}^{\mathcal{I}}, C_{U U}^{\mathcal{I}}$ terms (see Figs. 7 and 8).

(v) Finally, in Figs. 11 and 12, we present our results for the polarized beam cross section, $\sigma_{L U}$, Eq. (14). In Fig. 11, we show the comparison with BKM'01 and BKM'10. Notice that with the VA formalism one can see that the twist-two CFFs do not describe quantitatively the cross section at $Q^{2}=1.8 \mathrm{GeV}^{2}$, while the agreement improves increasing $Q^{2}$ to $4.5 \mathrm{GeV}^{2}$. Figure 12 displays the different contributions from the $A_{L U}^{\mathcal{I}}$, $B_{L U}^{\mathcal{I}}$, and $C_{L U}^{\mathcal{I}}$ terms, Eq. (19). Notice, in this case, the smallness of the axial contribution, $\propto G_{M} \Im m \widetilde{H}$.

\section{A. GPD Model}

To compare the VA and BKM cross section frameworks, we used the parametrization from Refs. [33,45,46] which is based on the Reggeized diquark model. In the DGLAP region, $x>\xi$, the parametric form for $F_{q}=H_{q}, E_{q}, \widetilde{H}_{q}, \widetilde{E}_{q}$, at the initial scale $Q_{o}^{2} \approx 0.1 \mathrm{GeV}^{2}$, reads 

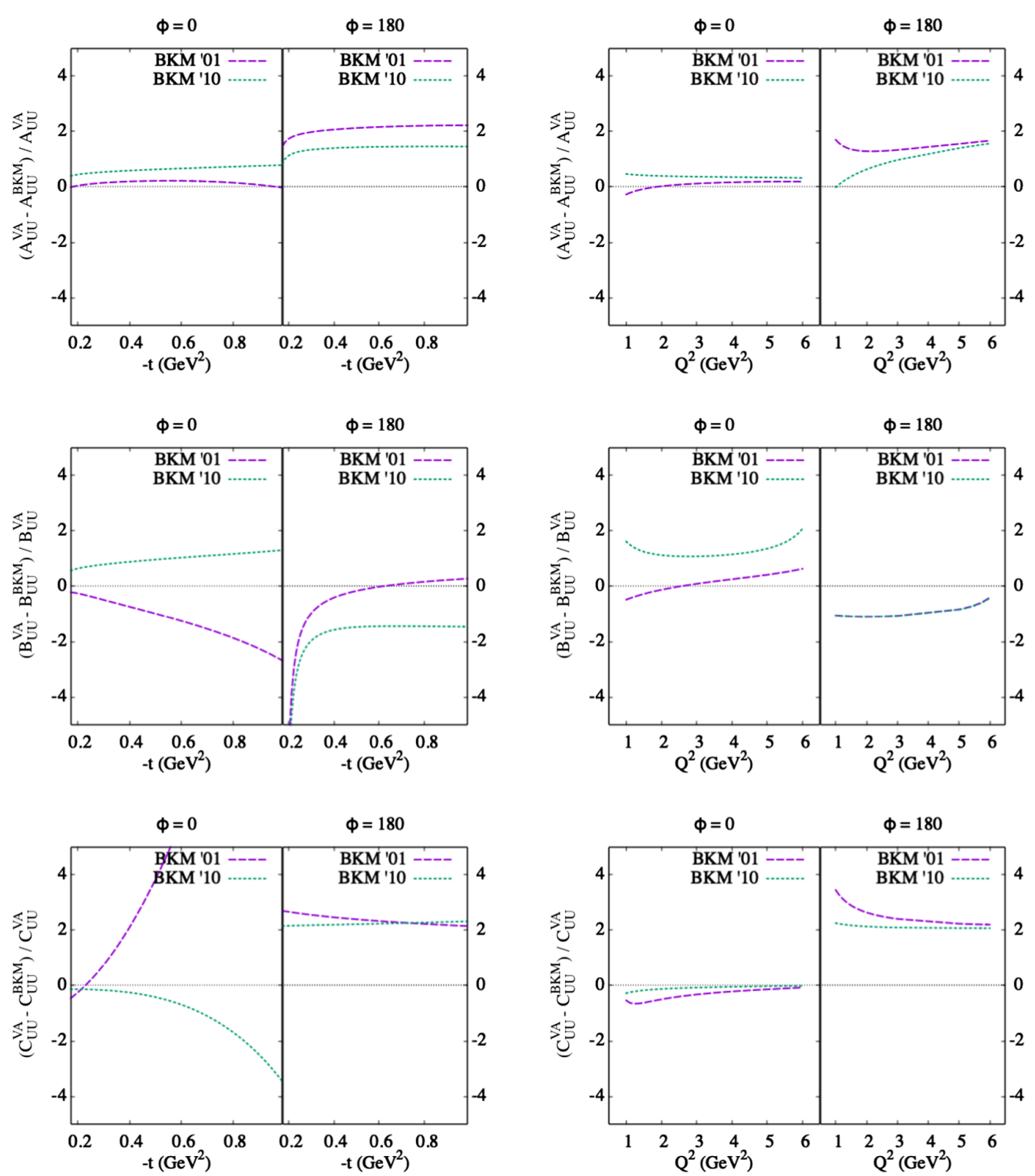

FIG. 9. Coefficients $A_{U U}^{\mathcal{I}}$ (top panels), $B_{U U}^{\mathcal{I}}$ (middle panels), and $C_{U U}^{\mathcal{I}}$ (bottom panels). On the lhs, the coefficients are compared to the formulation from Ref. [15] (BKM'01) and Ref. [16] (BKM'10), plotted vs $-t$ at two different values of $\phi$ for the kinematic setting: $\epsilon_{1}=5.7 \mathrm{GeV}, x_{B j}=0.34, Q^{2}=1.8 \mathrm{GeV}^{2}$. On the rhs, we show the percentage deviations between the BKM'01 and BKM'10 calculations and the formalism presented in this paper plotted vs $Q^{2}$, for $\epsilon_{1}=10.591 \mathrm{GeV}, x_{B j}=0.34,-t=0.17 \mathrm{GeV}^{2}$. 


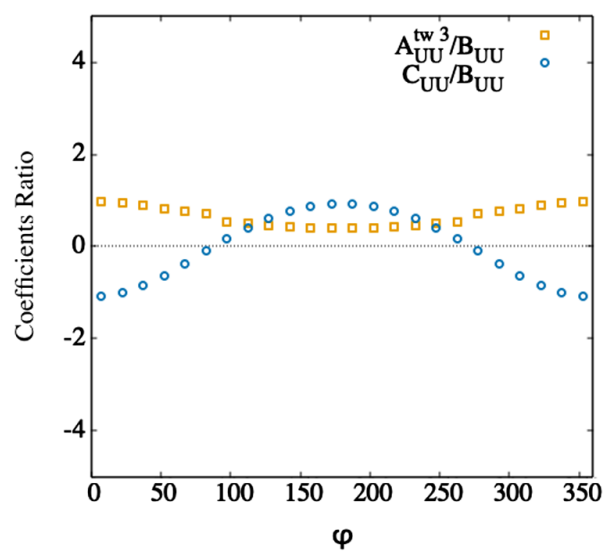

FIG. 10. Ratio of the kinematic coefficient of the twist-three term, $A_{U U}^{(3) \mathcal{I}}$, in Eq. (25), over the twist-two coefficient $B_{U U}$ in Eq. (18), plotted vs $\phi$ for $\epsilon_{1}=5.75 \mathrm{GeV}, Q^{2}=1.820 \mathrm{GeV}^{2}$, $x_{B j}=0.34$ and $t=-0.17 \mathrm{GeV}^{2}$. For comparison, we also plot the ratio of the kinematically subdominant twist-two coefficient, $C_{U U}$ over $B_{U U}$.

$F_{q}(x, \xi, t)=\mathcal{N}_{q} x^{\left[\alpha_{q}+\alpha_{q}^{\prime}(1-x)^{p q} t\right]} F_{\mathrm{diq}}(x, \xi, t), \quad q=u, d$,

where $F_{\text {diq }}$ is obtained from a diquark calculation with mass parameters, $m_{q}$ (quark mass), $M_{\Lambda}$ (dipole cut-off mass), $M_{X}^{q}$ (spectator diquark mass), and $\propto x^{\left[\alpha_{q}+\alpha_{q}^{\prime}(1-x)^{p} q\right]}$ accounts for the Regge behavior at low $x$. In the Efremov-Radyushkin-Brodsky-Lepage (ERBL) region, $-\xi<x<\xi$, we use a simple parametric form constrained by parity conservation and charge conjugation. The parameters for the twist-two GPDs are constrained from experimental data on the nucleon elastic form factors and PDFs, using

(i) the GPD normalization conditions,

$$
\begin{aligned}
\int_{-1}^{1} H_{q}\left(x, \xi, t ; Q^{2}\right) d x & =F_{1}^{q}(t), \\
\int_{-1}^{1} E_{q}\left(x, \xi, t ; Q^{2}\right) d x & =F_{2}^{q}(t), \\
\int_{-1}^{1} \widetilde{H}_{q}\left(x, \xi, t ; Q^{2}\right) d x & =G_{A}^{q}(t), \\
\int_{-1}^{1} \widetilde{E}_{q}\left(x, \xi, t ; Q^{2}\right) d x & =G_{P}^{q}(t),
\end{aligned}
$$

where we used the flavor separated data on the elastic nucleon form factors, $F_{1}^{q}$ and $F_{2}^{q}$ [50], and the
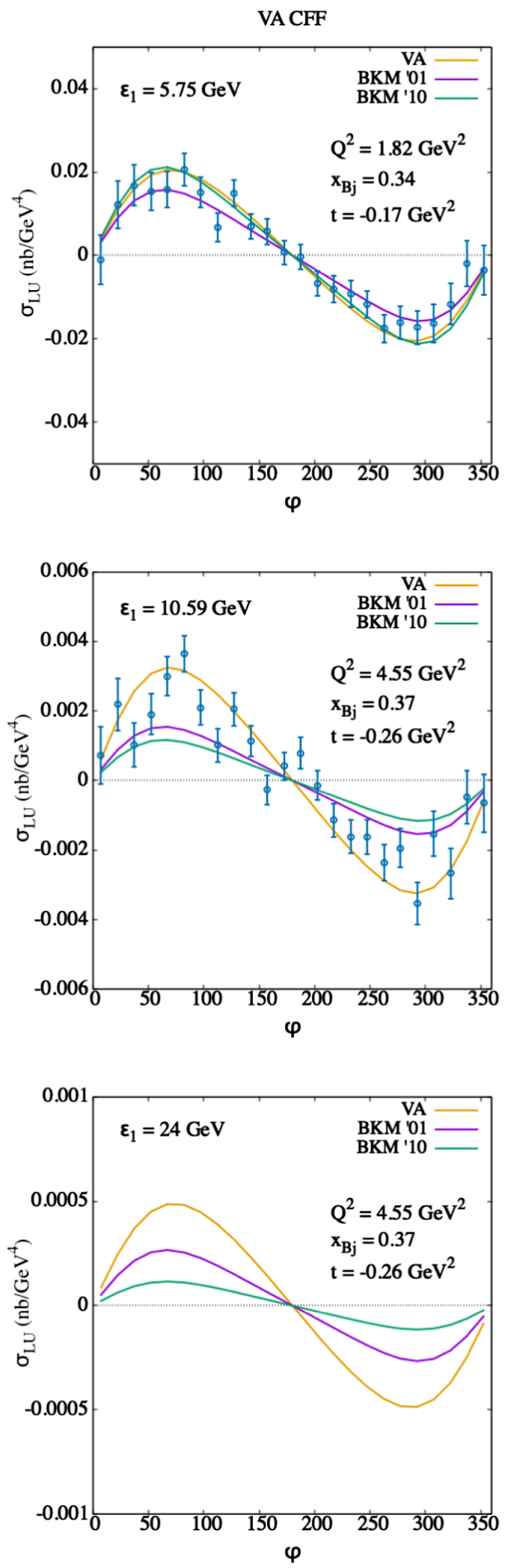

FIG. 11. Total LU cross section with VA theory CFFs at kinematic bins: $Q^{2}=1.82 \mathrm{GeV}^{2}, x_{B j}=0.34, t=-0.17 \mathrm{GeV}^{2}$, $\epsilon_{1}=5.75 \mathrm{GeV}$ (top); $Q^{2}=4.55 \mathrm{GeV}^{2}, x_{B j}=0.37, t=-0.26 \mathrm{GeV}^{2}$, $\epsilon_{1}=10.591 \mathrm{GeV} \quad$ (middle); $Q^{2}=4.55 \mathrm{GeV}^{2}, \quad x_{B j}=0.37$, $t=-0.26 \mathrm{GeV}^{2}, \epsilon_{1}=24 \mathrm{GeV}$ (bottom). 

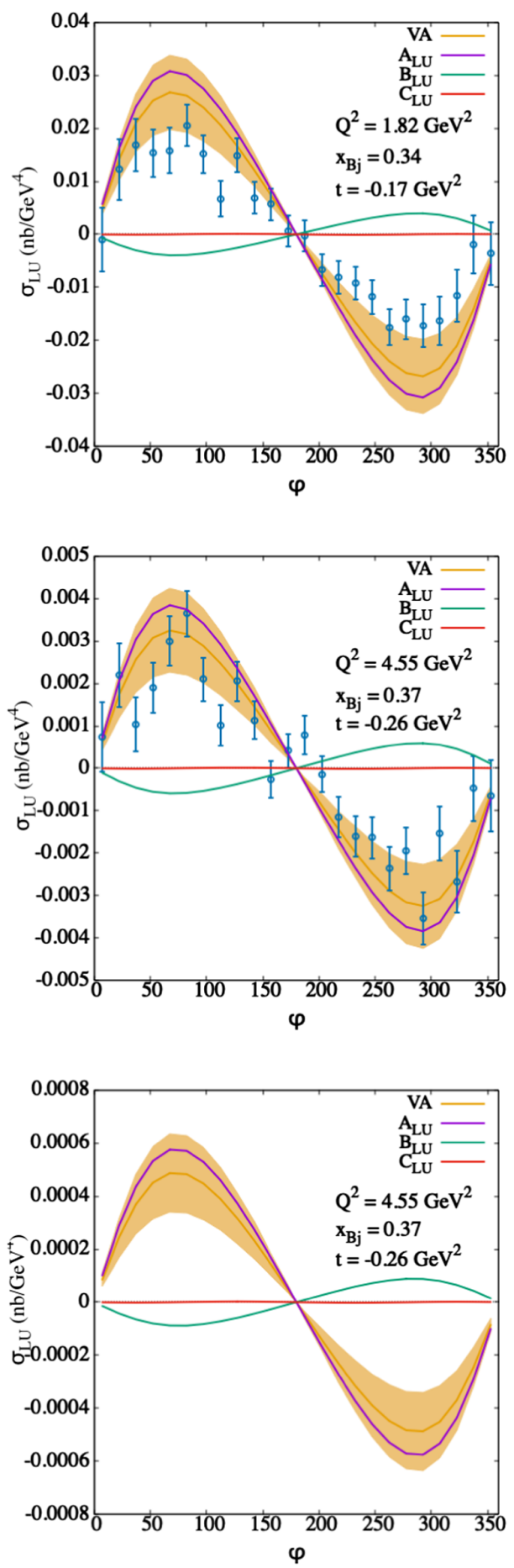

FIG. 12. Total LU cross section with VA theory CFFs at the same kinematic bins as Fig. 11. The different contributions from the $A_{L U}, B_{L U}$, and $C_{L U}$ terms are shown.

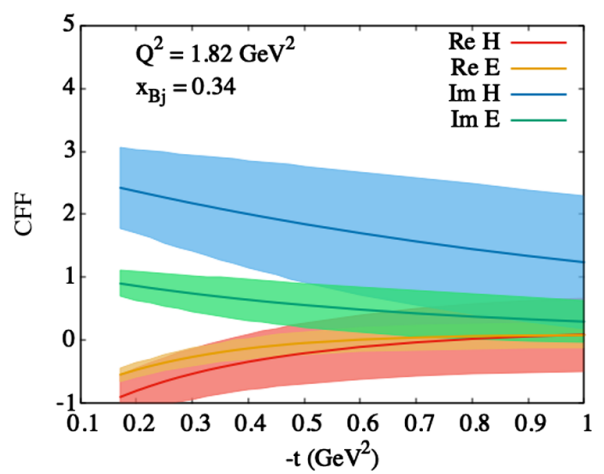

FIG. 13. VA Compton form factors $H$ and $E$ calculated in the Reggeized diquark model, Eq. (34).

nucleon axial [51] and pseudoscalar [52] form factor parametrizations,

(ii) the forward limit conditions,

$$
\begin{aligned}
H_{q}\left(x, 0,0 ; Q^{2}\right) & =q\left(x, Q^{2}\right), \widetilde{H}_{q}\left(x, 0,0 ; Q^{2}\right) \\
& =\Delta q\left(x, Q^{2}\right),
\end{aligned}
$$

with the unpolarized PDF, $q(x)$, and the helicity distribution, $\Delta q(x)$, being evaluated using current nucleon PDFs parametrizations (details are in Refs. [53,54]). To compare with data, the GPDs are perturbatively evolved at leading order to the scale, of the data, $Q^{2}[4,5,32,44]$.

In Table II, we present the values of the CFFs, $\mathcal{H}, \mathcal{E}, \widetilde{\mathcal{H}}$, $\widetilde{\mathcal{E}}$, calculated at $x_{B j}=0.34,0.37,-t=0.17,0.26 \mathrm{GeV}^{2}$, and $Q^{2}=1.8,4.5 \mathrm{GeV}^{2}$, compared with the values from the analyses in Refs. [48,49]. The imaginary and real components of $\mathcal{H}$ and $\mathcal{E}$ from the VA model are also shown in Fig. 13 in a similar kinematic range. The uncertainty bands in the figure represent the theoretical error of the parametrization [45].

In Fig. 14, we show the potential impact of the new formalism on extracting the values of the CFFs from experiment. On the rhs, we show $\sigma_{U U}^{\mathcal{I}}+\sigma_{U U}^{\mathrm{DVCS}}$ using the CFFs extracted from the fit in Ref. [49] using the BKM formalism. On the lhs, we show the same quantity evaluated using the same CFFs from Ref. [49], but with the VA formalism. The fact that the data on the lhs can no longer be fitted quantifies once more the discrepancies between the two frameworks. Notice, in particular, that the value of the KM15 $\Re e \widetilde{E}$ contributing 

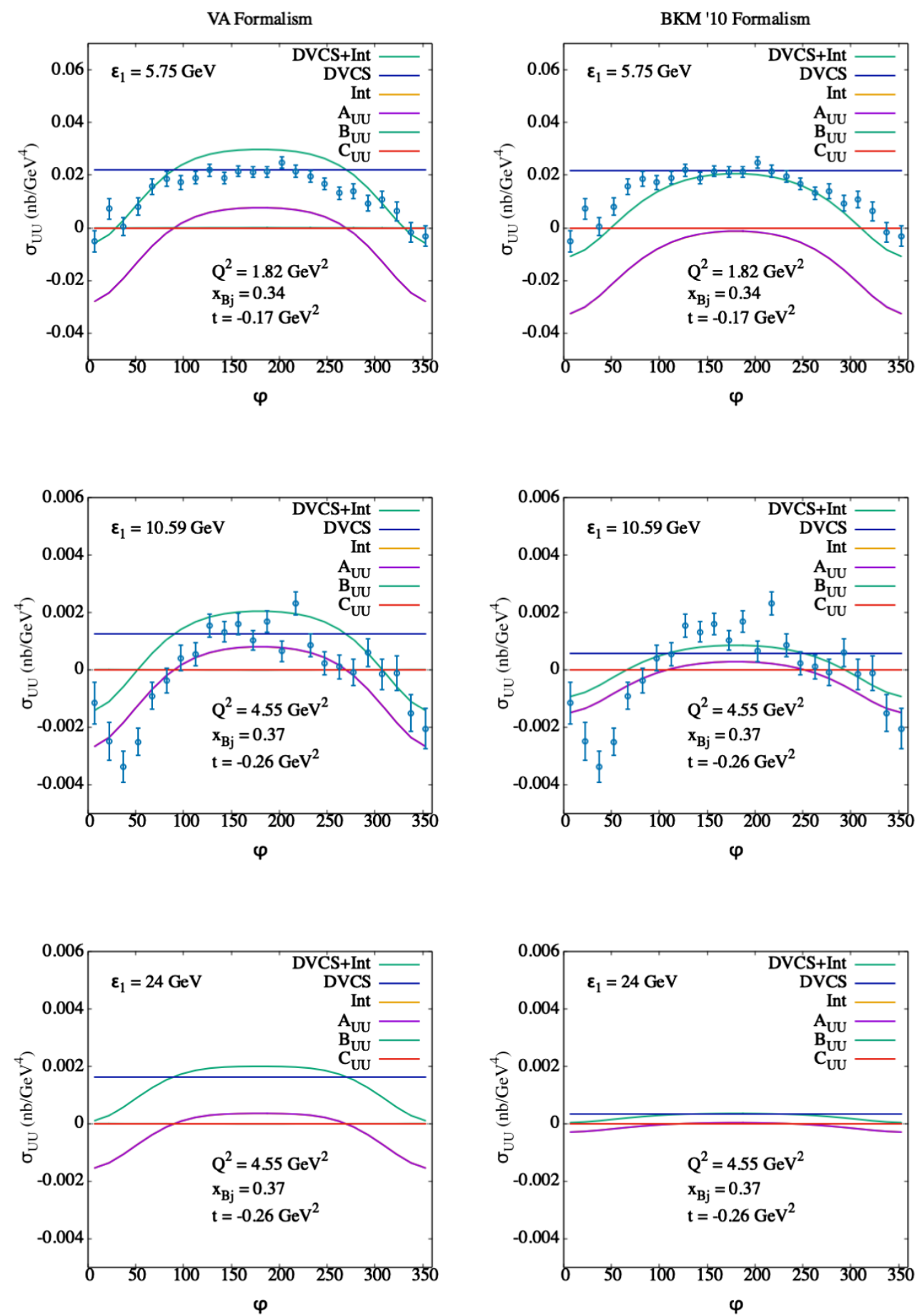

FIG. 14. Sum of contributions $\sigma_{U U}^{\mathcal{I}}+\sigma_{U U}^{\text {DVCS }}$ using CFF values from Kumericki et al. [49] in two different formalisms for the cross section: VA formalism (left) and BKM10 formalism (right). 

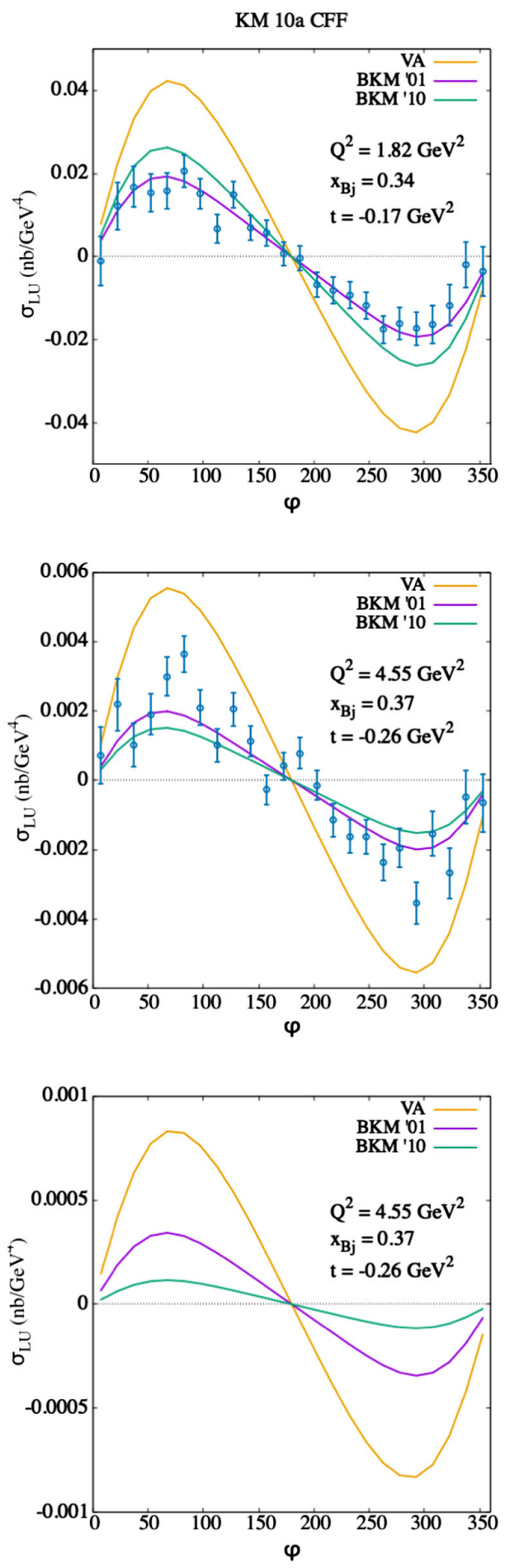

FIG. 15. Total LU cross section with Kumericki CFFs at kinematic bin. Left: $Q^{2}=1.82 \mathrm{GeV}^{2}, x_{B j}=0.34, t=-0.17 \mathrm{GeV}^{2}$, $\epsilon=5.75 \mathrm{GeV}$. Right: $Q^{2}=4.55 \mathrm{GeV}^{2}, \quad x_{B j}=0.37, \quad t=$ $-0.26 \mathrm{GeV}^{2}, \epsilon=10.591 \mathrm{GeV}$. Our prediction for a $24 \mathrm{GeV}$ beam energy. to $\sigma_{U U}^{\mathrm{DVCS}}$ makes this term three times larger than our value. (All values of the form factors used in the plots are displayed in Table II.) Figure 15 shows a similar trend for $\sigma_{L U}$.

\section{CONCLUSIONS AND OUTLOOK}

In order to extract information on the QCD matrix elements of deeply virtual exclusive electron scattering processes, one needs to first understand the detailed structure of the cross section. In deeply virtual exclusive photoproduction, in particular, tracking analytically the dependence in the high $Q^{2}$ limit on the invariants $x_{B j}$ and $t$, as well as on the angle $\phi$ between the lepton and hadron planes, has constituted a challenge which has been hampering, so far, a clean extraction of the various contributions to the cross section.

In our study of unpolarized scattering, differently from previous approaches, we argue that by organizing the cross section for the $e p \rightarrow e^{\prime} p^{\prime} \gamma$ scattering process according to its electromagnetic structure allows us to clearly separate the contributions of the various twist-two CFFs as well as the twist-three components.

Furthermore the cross section is described in terms of manageable and streamlined structures for the $\mathrm{BH}$ and DVCS contributions up to twist three. In particular, the DVCS-BH interference term is described by

(i) an electric contribution, $\left(F_{1}-\tau F_{2}\right)(\mathcal{H}-\tau \mathcal{E})$,

(ii) a magnetic contribution, $\left(F_{1}+F_{2}\right)(\mathcal{H}+\mathcal{E})$ containing the combination of GPDs necessary to extract angular momentum,

(iii) an axial contribution, $\left(F_{1}+F_{2}\right) \widetilde{\mathcal{H}}$.

The latter is reminiscent of the $G_{M} G_{A}$ contribution in elastic scattering, but it is now allowed without violating parity conservation because of the extra degree of freedom provided by the outgoing photon with momentum $q^{\prime} \neq q$.

The kinematic coefficients are lengthy but straightforward to calculate functions of $\phi$ evaluated to all orders in $1 / Q$. In addition to a kinematic $\phi$ dependence, we explain how the virtual photon phase $\phi$ dependence originates in a clearly distinguishable way. This distinction is important to for disentangling terms of different twist both in DVCS and in related processes including, e.g., timelike Compton scattering.

The reorganization of the cross section also uncovers substantial discrepancies with the harmonics decomposition of BKM. We find several discrepancies in both the pure DVCS and DVCS-BH interference terms, while the BH contribution turns out to be numerically equivalent. These discrepancies are important and can affect considerably the 
extraction of CFFs from data, and consequently, any conclusion on the behavior of angular momentum, pressure, or shear forces inside the proton. We tracked the differences between the BKM and VA formalism numerically, as a function of the various kinematic variables involved. We conclude that while the differences tend to be reduced at high $Q^{2}$ for some of the observables, this is not a general rule.

What is the inherent reason behind such discrepancies? Our results might be compared, in principle, with the studies in Refs. [23-25] which result in both $t$-dependent and target mass corrections, albeit using the harmonics decomposition. A common point of view is that the choice of the leading twist decomposition of the DVCS hadronic tensor is not unique. The consequences of this ambiguity results in a different structure of power corrections.

Future work in this direction, including numerical evaluations of twist-three three CFFs, as well as a straightforward extension of our framework to timelike Compton scattering, will help us determine unambiguously the internal dynamics and mechanical properties of the proton.

\section{ACKNOWLEDGMENTS}

We thank Southeastern Universities Research Association (SURA) and Jefferson Lab for the support from the Center for Nuclear Femtography and, in particular, to our colleagues participating in the University of Virginia lead initiative, Pete Alonzi, Matthias Burkardt (NMSU), Gordon Cates, Donal Day, and Joshua Hoskins. We are also grateful to Jian-Ping Chen, Xu Cao, and Yuxiang Zhao for discussing the projected EicC kinematic settings. Comments from Marie Boer, Markus Diehl, and Charles Hyde are also gratefully acknowledged. Finally, we thanks Kyle Shiells and Yushun Guo for a thorough check of many of our formulas. This work was funded by DOE Grant No. DE-SC0016286 and in part by the DOE Topical Collaboration on TMDs (B. K. and S. L.).

\section{APPENDIX A: CROSS SECTION PHASE SPACE FACTORS}

The cross section for BKM and in this paper (VA) are written in terms of different kinematic variables as

$$
\begin{gathered}
(\mathrm{VA}) \rightarrow \frac{d \sigma}{d x d Q^{2} d t d \phi}=\Gamma_{\mathrm{VA}}|T|^{2}, \\
(\mathrm{BKM}) \rightarrow \frac{d \sigma}{d x d y d t d \phi}=\Gamma_{\mathrm{BKM}}|T|^{2},
\end{gathered}
$$

where

$$
\begin{aligned}
\Gamma_{\mathrm{VA}} & =\frac{1}{\left(s-M^{2}\right)^{2} x}, \quad \Gamma_{\mathrm{BKM}}=\frac{x y}{Q^{2}}=\frac{1}{s-M^{2}}, \\
\frac{\Gamma_{\mathrm{VA}}}{\Gamma_{\mathrm{BKM}}} & =\frac{Q^{2}}{\left(s-M^{2}\right)^{2} x^{2} y}=\frac{y}{Q^{2}} .
\end{aligned}
$$

\section{APPENDIX B: KINEMATIC VARIABLES IN FIXED TARGET AND COLLIDER FRAMES}

To describe the reaction

$$
k+p \rightarrow k^{\prime}+p^{\prime}+q^{\prime}
$$

one defines the lepton plane through the four-momenta $k$ and $k^{\prime}$, with $q=k-k^{\prime}$, and the initial proton fourmomenta, $p$; the final photon momentum, $q^{\prime}$, and the final proton momentum, $p^{\prime}$ define the hadron scattering plane.

The cross section, Eq. (1), depends on the variables $k_{o}$ (initial electron energy), $Q^{2}, x_{B j}=Q^{2} / 2 M \nu$ ), $t$, and $\phi$.

\section{Laboratory frame}

In the lab, or proton rest frame, the initial electron is aligned with the $z$ axis, and the final electron is scattered in the $x--z$ plane with components

$$
\begin{aligned}
k & \equiv\left(k_{o} ; 0,0, k_{3}=-|\vec{k}| \approx-k_{o}\right), \\
p & \equiv(M ; 0,0,0) \\
k^{\prime} & \equiv\left(k_{o}^{\prime}=k_{o}-\frac{Q^{2}}{2 M x_{B j}} ; \sqrt{k_{o}^{\prime 2}-k_{3}^{\prime 2}}, 0, \frac{-Q^{2}+2 k_{o}^{\prime} k_{o}}{2 k_{3}}\right) \\
q & =k-k^{\prime} \equiv\left(\nu ;-\sqrt{k_{o}^{\prime 2}-k_{3}^{\prime 2}}, 0,-\nu\left(1+\frac{\gamma^{2}}{2 y}\right)\right),
\end{aligned}
$$

where, disregarding the electron mass, $k_{o}=|\vec{k}|=\epsilon_{1}$. The cross section calculations are done with $q$ aligned along the $z$ axis, i.e., rotating from the lab frame by the angle

$$
\alpha=\arctan \left(q_{1} / q_{3}\right)
$$

in the $x--z$ plane. The rotated four vectors in this frame are denoted by, $k^{R}, k^{\prime R}, q^{R}$.

The outgoing photon components are derived from the relations

$$
\begin{aligned}
p_{o}^{\prime}+q_{o}^{\prime} & =M+\nu, \\
p_{3}^{\prime R}+q_{3}^{\prime R} & =q_{3}^{R},
\end{aligned}
$$

and using the definitions

$$
\begin{aligned}
& t=\left(p-p^{\prime}\right)^{2}=2 M^{2}-2\left(p p^{\prime}\right), \\
& t=\left(q^{\prime}-q\right)^{2}=-Q^{2}-2\left(q q^{\prime}\right)
\end{aligned}
$$


one obtains

$$
q^{\prime R} \equiv\left(q_{o}^{\prime} ; q_{o}^{\prime} \sin \theta \cos \phi, q_{o}^{\prime} \sin \theta \sin \phi, q_{3}^{\prime R}=q_{o}^{\prime} \cos \theta\right),
$$

where

$$
\begin{aligned}
q_{o}^{\prime} & =\nu+\frac{t}{2 M}, \quad q_{3}^{\prime R}=-\frac{t+Q^{2}+2 q_{o} q_{o}^{\prime}}{2\left|\vec{q}^{R}\right|}, \\
\theta & =\arccos \left(q_{3}^{\prime R} / q_{o}^{\prime}\right) .
\end{aligned}
$$

From these vectors, one defines $\Delta=q-q^{\prime}$ and $p^{\prime}=$ $p+\Delta$.

\section{Collider frame}

In the collider frame, with the initial proton moving along the positive $z$ axis, one has

$$
\begin{aligned}
k & \equiv\left(k_{o} ; 0,0, k_{3}=-|\vec{k}| \approx-k_{o}\right), \\
p & \equiv\left(p_{o}=\sqrt{p_{3}+M^{2}} ; 0,0, p_{3}\right), \\
k^{\prime} & \equiv\left(k_{o}^{\prime} ; \sqrt{k_{o}^{\prime 2}-k_{3}^{\prime 2}}, 0, \frac{-Q^{2}+2 k_{o}^{\prime} k_{o}}{2 k_{3}}\right), \\
q & =k-k^{\prime},
\end{aligned}
$$

with

$$
\begin{aligned}
k_{o}^{\prime}= & k_{o}-\left[2 \widetilde{\nu}-\sqrt{\widetilde{\nu}^{2}-4\left(1-\left(\frac{p_{3}}{p_{o}}\right)^{2}\right)\left(\widetilde{\nu}^{2}-\frac{p_{3}}{p_{o}} Q^{2}\right)}\right] \\
& \times\left[2\left(1-\left(\frac{p_{3}}{p_{o}}\right)\right]^{-1}\right. \\
\approx & k_{o}-\frac{\widetilde{\nu}}{2}+p_{o} x_{B j}, \\
\widetilde{\nu} & =\frac{Q^{2}}{2 p_{o} x_{B j}} .
\end{aligned}
$$

The outgoing photon four-momenta components are derived by first boosting to the c.m. frame with

$$
\beta=-\frac{p_{3}}{p_{o}}, \quad \gamma=\frac{p_{o}}{M},
$$

so that the components of the boosted virtual photon $q^{B}$ are

$$
\begin{aligned}
q_{o}^{B} & =\gamma\left(q_{o}+\beta q_{3}\right), \\
q_{1,2}^{B} & =q_{1,2}, \\
q_{3}^{B} & =\gamma\left(q_{3}+\beta q_{o}\right) .
\end{aligned}
$$

One now rotates $q^{B}$ to obtain a vector $q^{R}$ aligned with the $z$ axis using

$$
\alpha=\arctan \left(q_{1}^{B} / q_{3}^{B}\right) .
$$

The outgoing photon has components

$$
q^{\prime R} \equiv\left(q_{o}^{\prime} ; q_{o}^{\prime} \sin \theta \cos \phi, q_{o}^{\prime} \sin \theta \sin \phi, q_{3}^{\prime R}\right),
$$

where

$$
\begin{aligned}
q_{o}^{\prime} & =q_{o}^{B}+\frac{t}{2 M}, \quad q_{3}^{\prime R}=-\frac{t+Q^{2}+2 q_{o}^{B} q_{o}^{\prime}}{2\left|\vec{q}^{B}\right|}, \\
\theta & =\arccos \left(q_{3}^{\prime R} / q_{o}^{\prime}\right) .
\end{aligned}
$$

$\Delta$ and $p^{\prime}$ are reconstructed from these vectors.

\section{APPENDIX C: KINEMATIC COEFFICIENTS FOR BH and BH-DVCS INTERFERENCE TERMS}

For convenience, we write the expressions of the kinematic coefficients relevant for this paper. All coefficients were calculated in Ref. [9] in a covariant form using four-vector products with notation, $(a b)=a_{o} b_{o}-\vec{a} \cdot \vec{b}$. The relevant four-vector components for both the laboratory frame and the collider frame are given in Appendix B.

The BH coefficients in Eq. (15) read

$$
\begin{gathered}
A_{\mathrm{BH}}=\frac{8 M^{2}}{t\left(k q^{\prime}\right)\left(k^{\prime} q^{\prime}\right)}\left[4 \tau\left((k P)^{2}+\left(k^{\prime} P\right)^{2}\right)-(\tau+1)\left((k \Delta)^{2}+\left(k^{\prime} \Delta\right)^{2}\right)\right], \\
B_{\mathrm{BH}}=\frac{16 M^{2}}{t\left(k q^{\prime}\right)\left(k^{\prime} q^{\prime}\right)}\left[(k \Delta)^{2}+\left(k^{\prime} \Delta\right)^{2}\right],
\end{gathered}
$$

where $P=\left(p+p^{\prime}\right) / 2, \tau=-t / 4 M^{2}$. 
The BH-DVCS interference cross section coefficients in Eq. (18) read

$$
\begin{aligned}
A_{U U}^{\mathcal{I}}= & 2\left(P \Sigma_{S}^{\cos \phi}\right) \\
= & -8 D_{+}\left[\left(k^{\prime} P\right)\left(2 k_{T}^{2}-k_{T} \cdot q_{T}^{\prime}-2\left(k q^{\prime}\right)\right)+(k P)\left(2 k_{T}^{\prime} \cdot k_{T}+k_{T}^{\prime} \cdot q_{T}^{\prime}+2\left(k^{\prime} q^{\prime}\right)\right)-\left(k_{T} \cdot P_{T}\right)\left(2\left(k k^{\prime}\right)+\left(k^{\prime} q^{\prime}\right)\right)\right. \\
& \left.+\left(k_{T}^{\prime} \cdot P_{T}\right)\left(k q^{\prime}\right)\right] \cos \phi-8 D_{-}\left[\left(P q^{\prime}\right)\left(2 k_{T} \cdot k_{T}^{\prime}+2\left(k k^{\prime}\right)\right)-\left(k_{T} \cdot P_{T}\right)\left(k^{\prime} q^{\prime}\right)-\left(k_{T}^{\prime} \cdot P_{T}\right)\left(k q^{\prime}\right)\right. \\
& \left.+\left(P_{T} \cdot q_{T}^{\prime}\right)\left(k k^{\prime}\right)\right] \cos \phi, \\
B_{U U}^{\mathcal{I}}= & \xi\left(\Delta \Sigma_{S}^{\cos \phi}\right) \\
= & -4 \xi D_{+}\left[\left(k^{\prime} \Delta\right)\left(2 k_{T}^{2}-k_{T} \cdot q_{T}^{\prime}-2\left(k q^{\prime}\right)\right)+(k \Delta)\left(2 k_{T}^{\prime} \cdot k_{T}+k_{T}^{\prime} \cdot q_{T}^{\prime}+2\left(k^{\prime} q^{\prime}\right)\right)-\left(k_{T} \cdot \Delta_{T}\right)\left(2\left(k k^{\prime}\right)+\left(k^{\prime} q^{\prime}\right)\right)\right. \\
& \left.+\left(k_{T}^{\prime} \cdot \Delta_{T}\right)\left(k q^{\prime}\right)\right] \cos \phi-4 \xi D_{-}\left[\left(\Delta q^{\prime}\right)\left(2 k_{T} \cdot k_{T}^{\prime}+2\left(k k^{\prime}\right)\right)-\left(k_{T} \cdot \Delta_{T}\right)\left(k^{\prime} q^{\prime}\right)-\left(k_{T}^{\prime} \cdot \Delta_{T}\right)\left(k q^{\prime}\right)\right. \\
& \left.+\left(q_{T}^{\prime} \cdot \Delta_{T}\right)\left(k k^{\prime}\right)\right] \cos \phi, \\
& \quad C_{U U}^{\mathcal{I}}=\frac{1}{2 P^{+}} \epsilon^{\mu \sigma \nu+}\left(P_{\mu} \Delta_{\nu}-P_{\nu} \Delta_{\mu}+\frac{1}{2} \Delta_{\mu} \Delta_{\nu}\right) g_{\sigma \rho}\left(\Sigma_{A}^{\cos \phi}\right)^{\rho} \\
= & 4 \frac{D_{+}}{P^{+}}\left[-\left(k_{T} \cdot P_{T}\right)\left(q_{T}^{\prime} \cdot \Delta_{T}\right) k^{\prime+}+\left(k_{T} \cdot \Delta_{T}\right)\left(P_{T} \cdot q_{T}^{\prime}\right) k^{\prime+}+\left(k_{T}^{\prime} \cdot P_{T}\right)\left(q_{T}^{\prime} \cdot \Delta_{T}\right) k^{+}\right. \\
& \quad-\left(k_{T}^{\prime} \cdot \Delta_{T}\right)\left(P_{T} \cdot q_{T}^{\prime}\right) k^{+}-2\left(k k^{\prime}\right)\left(k_{T}^{\prime} \cdot P_{T}\right) \Delta^{+}-\left(k q^{\prime}\right)\left(k_{T}^{\prime} \cdot P_{T}\right) \Delta^{+} \\
& \left.+\left(k^{\prime} q^{\prime}\right)\left(k_{T} \cdot P_{T}\right) \Delta^{+}+2\left(k k^{\prime}\right)\left(k_{T}^{\prime} \cdot \Delta_{T}\right) P^{+}+\left(k q^{\prime}\right)\left(k_{T}^{\prime} \cdot \Delta_{T}\right) P^{+}-\left(k^{\prime} q^{\prime}\right)\left(k_{T} \cdot \Delta_{T}\right) P^{+}\right] \cos \phi \\
& +4 \frac{D_{-}}{P^{+}}\left[\left(k^{\prime} q^{\prime}\right)\left(k_{T} \cdot P_{T}\right) \Delta^{+}-\left(k k^{\prime}\right)\left(q_{T}^{\prime} \cdot P_{T}\right) \Delta^{+}+\left(k q^{\prime}\right)\left(k_{T}^{\prime} \cdot P_{T}\right) \Delta^{+}\right. \\
& \left.\quad-\left(k^{\prime} q^{\prime}\right)\left(k_{T} \cdot \Delta_{T}\right) P^{+}+\left(k k^{\prime}\right)\left(q_{T}^{\prime} \cdot \Delta_{T}\right) P^{+}-\left(k q^{\prime}\right)\left(k_{T}^{\prime} \cdot \Delta_{T}\right) P^{+}\right] \cos \phi,
\end{aligned}
$$

where $\left(\Sigma_{S}^{\cos \phi}\right)^{\rho}$ and $\left(\Sigma_{A}^{\cos \phi}\right)^{\rho}$ are linear combinations of the kinematic four vectors, $k^{\rho}, k^{\prime \rho}, q^{\prime \rho}$, describing the BH-DVCS interference lepton tensor [9],

$$
\begin{aligned}
\left(\Sigma_{S}^{\cos \phi}\right)^{\rho}= & \left\{k^{\prime \rho}\left[\left(2 k_{\mu} k_{\nu}-q_{\mu}^{\prime} k_{\nu}\right) g_{T}^{\mu \nu}+2\left(k q^{\prime}\right)\right] 2 D_{+}+k^{\rho}\left[\left(2 k_{\mu}^{\prime} k_{\nu}+q_{\mu}^{\prime} k_{\nu}^{\prime}\right) g_{T}^{\mu \nu}-2\left(k^{\prime} q^{\prime}\right)\right] 2 D_{+}\right. \\
& +q^{\rho \rho}\left[2 k_{\mu} k_{\nu}^{\prime} g_{T}^{\mu \nu}-2\left(k k^{\prime}\right)\right] D_{-}+g_{T}^{\rho \mu}\left[\left(-2\left(k k^{\prime}\right)+\left(k^{\prime} q^{\prime}\right)\right) k_{\mu}-\left(k q^{\prime}\right) k_{\mu}^{\prime}\right] 2 D_{+} \\
& \left.+g_{T}^{\rho \mu}\left[\left(k k^{\prime}\right) q_{\mu}^{\prime}-\left(k^{\prime} q^{\prime}\right) k_{\mu}-\left(k q^{\prime}\right) k_{\mu}^{\prime}\right] 2 D_{-}\right\} \cos \phi
\end{aligned}
$$

and

$$
\begin{aligned}
\left(\Sigma_{A}^{\cos \phi}\right)^{\rho}= & \left\{k^{\prime \rho}\left[\left(2 k_{\mu} k_{\nu}-q_{\mu}^{\prime} k_{\nu}\right) \epsilon_{T}^{\mu \nu}+2\left(k q^{\prime}\right)\right] 2 D_{+}+k^{\rho}\left[\left(2 k_{\mu}^{\prime} k_{\nu}+q_{\mu}^{\prime} k_{\nu}^{\prime}\right) \epsilon_{T}^{\mu \nu}-2\left(k^{\prime} q^{\prime}\right)\right] 2 D_{+}\right. \\
& +q^{\rho \rho}\left[2 k_{\mu} k_{\nu}^{\prime} \epsilon_{T}^{\mu \nu}-2\left(k k^{\prime}\right)\right] D_{-}+\epsilon_{T}^{\rho \mu}\left[\left(-2\left(k k^{\prime}\right)+\left(k^{\prime} q^{\prime}\right)\right) k_{\mu}-\left(k q^{\prime}\right) k_{\mu}^{\prime}\right] 2 D_{+} \\
& \left.+\epsilon_{T}^{\rho \mu}\left[\left(k k^{\prime}\right) q_{\mu}^{\prime}-\left(k^{\prime} q^{\prime}\right) k_{\mu}-\left(k q^{\prime}\right) k_{\mu}^{\prime}\right] 2 D_{-}\right\} \cos \phi .
\end{aligned}
$$

$D^{+}$and $D^{-}$are defined as

$$
D^{+}=\frac{\left(k q^{\prime}\right)-\left(k q^{\prime}\right)}{2\left(k^{\prime} q^{\prime}\right)\left(k q^{\prime}\right)}, \quad D^{-}=-\frac{\left(k q^{\prime}\right)+\left(k q^{\prime}\right)}{2\left(k^{\prime} q^{\prime}\right)\left(k q^{\prime}\right)}
$$

We also calculate leading terms that restore electromagnetic gauge invariance. We introduce these expressions below:

$$
A_{U U}^{\mathcal{I}}=A_{U U}^{\mathcal{I}}-8 D_{-}\left[\left(P k^{\prime}\right)\left(k_{T} \cdot q_{T}^{\prime}\right)+(P k)\left(k_{T}^{\prime} \cdot q_{T}^{\prime}\right)-\left(k k^{\prime}\right)\left(P_{T} \cdot q_{T}^{\prime}\right)\right] \cos \phi
$$




$$
\begin{aligned}
B_{U U}^{\mathcal{I}}= & B_{U U}^{\mathcal{I}}-4 \xi D_{-}\left[\left(k^{\prime} \Delta\right)\left(k_{T} \cdot q_{T}^{\prime}\right)+(k \Delta)\left(k_{T}^{\prime} \cdot q_{T}^{\prime}\right)-\left(k k^{\prime}\right)\left(q_{T}^{\prime} \cdot \Delta_{T}\right)\right] \cos \phi \\
& -2 \frac{t}{P^{+}} D_{+}\left[k^{\prime+}\left(2\left(k_{T} \cdot k_{T}\right)-\left(k_{T} \cdot q_{T}^{\prime}\right)-2\left(k q^{\prime}\right)\right)+k^{+}\left(2\left(k_{T}^{\prime} \cdot k_{T}^{\prime}\right)+\left(k_{T}^{\prime} \cdot q_{T}^{\prime}\right)+2\left(k^{\prime} q^{\prime}\right)\right)\right] \cos \phi \\
& -2 \frac{t}{P^{+}} D_{-}\left[q^{\prime+}\left(2\left(k_{T} \cdot k_{T}^{\prime}\right)+2\left(k k^{\prime}\right)\right)+k^{\prime+}\left(k_{T} \cdot q_{T}^{\prime}\right)+k^{+}\left(k_{T}^{\prime} \cdot q_{T}^{\prime}\right)\right] \cos \phi . \\
C_{U U}^{\mathcal{I} \prime}= & C_{U U}^{\mathcal{I}}+4 \frac{D_{-}}{P^{+}}\left[-2\left(P_{T} \cdot q_{T}^{\prime}\right)\left(k_{T} \cdot k_{T}^{\prime}\right) \Delta^{+}+\left(P_{T} \cdot q_{T}^{\prime}\right)\left(k_{T} \cdot \Delta_{T}\right) k^{\prime+}+\left(P_{T} \cdot q_{T}^{\prime}\right)\left(k_{T}^{\prime} \cdot \Delta_{T}\right) k^{+}\right. \\
& +2\left(k_{T}^{\prime} \cdot q_{T}^{\prime}\right)\left(k_{T} \cdot P_{T}\right) \Delta^{+}-2\left(k_{T}^{\prime} \cdot q_{T}^{\prime}\right)\left(k_{T} \cdot \Delta_{T}\right) P^{+}-\left(q_{T}^{\prime} \cdot \Delta_{T}\right)\left(k_{T} \cdot P_{T}\right) k^{\prime+}-\left(q_{T}^{\prime} \cdot \Delta_{T}\right)\left(P_{T} \cdot k_{T}^{\prime}\right) k^{+} \\
& \left.+2\left(q_{T}^{\prime} \cdot \Delta_{T}\right)\left(k_{T} \cdot k_{T}^{\prime}\right) P^{+}-\left(k k^{\prime}\right)\left(P_{T} \cdot q_{T}^{\prime}\right) \Delta^{+}+\left(k k^{\prime}\right)\left(q_{T}^{\prime} \cdot \Delta_{T}\right) P^{+}\right] \cos \phi .
\end{aligned}
$$

The coefficients $A_{U U}^{\mathcal{I}}, B_{U U}^{\mathcal{I}}$, and $C_{U U}^{\mathcal{I}}$ can be reevalauted using the expressions in BKM [15],

$$
\begin{gathered}
A_{U U}^{\mathcal{I}}=\frac{1}{x_{B j} y^{3} t \mathcal{P}_{1}(\phi) \mathcal{P}_{2}(\phi)}\left[-8 \frac{(2-y)^{3}}{1-y} K^{2}-8(2-y) \frac{t}{Q^{2}}(1-y)\left(2-x_{B j}\right)-8 K\left(2-2 y+y^{2}\right) \cos \phi\right], \\
B_{U U}^{\mathcal{I}}=\frac{\xi^{2}}{x_{B j} y^{3} t \mathcal{P}_{1}(\phi) \mathcal{P}_{2} \phi}\left[8(2-y) \frac{t}{Q^{2}}(1-y)\left(2-x_{B j}\right)\right], \\
C_{U U}^{\mathcal{I}}=\frac{\xi}{x_{B j} y^{3} t \mathcal{P}_{1}(\phi) \mathcal{P}_{2}(\phi)}\left[8 \frac{(2-y)^{3}}{(1-y)} K^{2}+8 K\left(2-y+y^{2}\right) \cos \phi\right],
\end{gathered}
$$

where

$$
\begin{gathered}
\mathcal{P}_{1}(\phi)=-\frac{1}{y\left(1+\gamma^{2}\right)}\{J+2 K \cos \phi\}, \quad \mathcal{P}_{2}(\phi)=1+\frac{t}{Q^{2}}+\frac{1}{y\left(1+\gamma^{2}\right)}\{J+2 K \cos \phi\}, \\
K^{2}=-\frac{t}{Q^{2}}\left(1-x_{B j}\right)\left(1-y-\frac{y^{2} \gamma^{2}}{4}\right)\left(1-\frac{t_{0}}{t}\right)\left(\sqrt{1+\gamma^{2}}+\frac{4 x_{B j}\left(1-x_{B j}\right)+\gamma^{2}}{4\left(1-x_{B j}\right)} \frac{t-t_{0}}{Q^{2}}\right), \\
J=\left(1-y-\frac{y \gamma^{2}}{2}\right)\left(1+\frac{t}{Q^{2}}\right)-\left(1-x_{B j}\right)(2-y) \frac{t}{Q^{2}},
\end{gathered}
$$

with $y=Q^{2} /\left(x_{B j}\left(s-M^{2}\right)\right), \gamma^{2}=4 M^{2} x_{B j}^{2} / Q^{2}, t_{o}=-2 \xi M^{2} /\left(1-\xi^{2}\right), \xi=x_{B j} /\left(2-x_{B j}\right)$.

The updated "BKM'10" expressions accounting for $1 / Q^{2}$ power corrections are given in the following form [16]:

$$
\begin{gathered}
A_{U U}^{\mathcal{I}}=\frac{1}{x_{B j} y^{3} t \mathcal{P}_{1}(\phi) \mathcal{P}_{2}(\phi)}\left\{\sum_{n=0}^{3} C_{++}^{\mathrm{unp}}(n) \cos (n \phi)\right\}, \\
B_{U U}^{\mathcal{I}}=\frac{\xi}{x_{B j} y^{3} t \mathcal{P}_{1}(\phi) \mathcal{P}_{2}(\phi)}\left\{\sum_{n=0}^{3} C_{++}^{\mathrm{unp}, V}(n) \cos (n \phi)\right\}, \\
C_{U U}^{\mathcal{I}}=\frac{-\xi}{x_{B j} y^{3} t \mathcal{P}_{1}(\phi) \mathcal{P}_{2}(\phi)}\left\{\sum_{n=0}^{3}\left(C_{++0}^{\mathrm{unp}, \mathrm{A}}(n)+C_{++}^{\mathrm{unp}}(n)\right) \cos (n \phi)\right\},
\end{gathered}
$$

where the expressions for $C_{++}^{\mathrm{unp}}(n), C_{++}^{\mathrm{unp}, \mathrm{V}}(n)$, and $C_{++0}^{\mathrm{unp}, \mathrm{A}}(n)$ can be found in Ref. [16].

The coefficients for the longitudinally polarized electron beam, Eq. (19), are the same as for the unpolarized case: $A_{L U}^{\mathcal{I}}=A_{U U}^{\mathcal{I}}, B_{L U}^{\mathcal{I}}=B_{U U}^{\mathcal{I}}$, and $C_{L U}^{\mathcal{I}}=C_{U U}^{\mathcal{I}}$. 
[1] X.-D. Ji, Phys. Rev. Lett. 78, 610 (1997).

[2] X.-D. Ji, Phys. Rev. D 55, 7114 (1997).

[3] J. C. Collins and A. Freund, Phys. Rev. D 59, 074009 (1999).

[4] X.-D. Ji and J. Osborne, Phys. Rev. D 57, R1337 (1998).

[5] X.-D. Ji and J. Osborne, Phys. Rev. D 58, 094018 (1998).

[6] M. Diehl, Phys. Rep. 388, 41 (2003).

[7] A. Belitsky and A. Radyushkin, Phys. Rep. 418, 1 (2005).

[8] K. Kumericki, S. Liuti, and H. Moutarde, Eur. Phys. J. A 52, 157 (2016).

[9] B. Kriesten, S. Liuti, L. Calero-Diaz, D. Keller, A. Meyer, G. R. Goldstein, and J. Osvaldo Gonzalez-Hernandez, Phys. Rev. D 101, 054021 (2020).

[10] C. Perdrisat, V. Punjabi, and M. Vanderhaeghen, Prog. Part. Nucl. Phys. 59, 694 (2007).

[11] H.-y. Gao, Int. J. Mod. Phys. E12, 1 (2003); E12, 567(E) (2003).

[12] T. Arens, O. Nachtmann, M. Diehl, and P. V. Landshoff, Z. Phys. C 74, 651 (1997).

[13] M. Diehl and S. Sapeta, Eur. Phys. J. C 41, 515 (2005).

[14] A. V. Belitsky, D. Mueller, L. Niedermeier, and A. Schafer, Nucl. Phys. B593, 289 (2001).

[15] A. V. Belitsky, D. Mueller, and A. Kirchner, Nucl. Phys. B629, 323 (2002).

[16] A. V. Belitsky and D. Mueller, Phys. Rev. D 82, 074010 (2010).

[17] A. V. Belitsky, D. Müller, and Y. Ji, Nucl. Phys. B878, 214 (2014).

[18] M. Burkardt, C. Miller, and W. Nowak, Rep. Prog. Phys. 73, 016201 (2010).

[19] B. Kriesten and S. Liuti, arXiv:2011.04484.

[20] P. A. M. Guichon and M. Vanderhaeghen, Prog. Part. Nucl. Phys. 41, 125 (1998).

[21] M. Vanderhaeghen, P. A. Guichon, and M. Guidal, Phys. Rev. Lett. 80, 5064 (1998).

[22] M. Vanderhaeghen, P. Guichon, and M. Guidal, Nucl. Phys. A654, 602c (1999).

[23] V. Braun and A. Manashov, J. High Energy Phys. 01 (2012) 085.

[24] V. Braun, A. Manashov, and B. Pirnay, Phys. Rev. Lett. 109, 242001 (2012).

[25] V. M. Braun, A. N. Manashov, D. Müller, and B. M. Pirnay, Phys. Rev. D 89, 074022 (2014).

[26] A. Bacchetta, M. Diehl, K. Goeke, A. Metz, P. J. Mulders, and M. Schlegel, J. High Energy Phys. 02 (2007) 093.

[27] A. Bogasz, https://www.jlab.org/accelerator-seminar-alexbogacz-remote (2021).

[28] D. P. Anderle et al., Front. Phys. (Beijing) 16, 64701 (2021).
[29] R. A. Khalek et al., arXiv:2103.05419.

[30] V. Dmitrasinovic and F. Gross, Phys. Rev. C 40, 2479 (1989); 43, 1495(E) (1991).

[31] S. Boffi, C. Giusti, and F. D. Pacati, Phys. Rep. 226, 1 (1993).

[32] K. J. Golec-Biernat and A. D. Martin, Phys. Rev. D 59, 014029 (1998).

[33] G. R. Goldstein, J. O. G. Hernandez, and S. Liuti, J. Phys. G 39, 115001 (2012).

[34] S. Meissner, A. Metz, and M. Schlegel, J. High Energy Phys. 08 (2009) 056.

[35] A. Rajan, M. Engelhardt, and S. Liuti, Phys. Rev. D 98, 074022 (2018).

[36] A. Rajan, A. Courtoy, M. Engelhardt, and S. Liuti, Phys. Rev. D 94, 034041 (2016).

[37] R. Jaffe and X.-D. Ji, Nucl. Phys. B375, 527 (1992).

[38] P. Mulders and R. Tangerman, Nucl. Phys. B461, 197 (1996).

[39] T. W. Donnelly and A. S. Raskin, Ann. Phys. (N.Y.) 169, 247 (1986).

[40] R. Gastmans and T. Wu, The Ubiquitos Photon (Clarendon Press, Oxford, 1990).

[41] M. Defurne et al., Nat. Commun. 8, 1408 (2017).

[42] F. Georges, Deeply virtual Compton scattering at Jefferson Lab, Ph.D. thesis, Institut de Physique Nucléaire d'Orsay, France, 2018.

[43] M. Defurne et al. (Jefferson Lab Hall A Collaboration), Phys. Rev. C 92, 055202 (2015).

[44] I. Musatov and A. Radyushkin, Phys. Rev. D 61, 074027 (2000).

[45] J. O. Gonzalez-Hernandez, S. Liuti, G. R. Goldstein, and K. Kathuria, Phys. Rev. C 88, 065206 (2013).

[46] B. Kriesten, P. Velie, E. Yeats, F. Y. Lopez, and S. Liuti, arXiv:2101.01826.

[47] G. R. Goldstein, J. O. Gonzalez-Hernandez, and S. Liuti, Phys. Rev. D 84, 034007 (2011).

[48] K. s. Kumerički and D. Müller, EPJ Web Conf. 112, 01012 (2016).

[49] K. Kumericki, T. Lautenschlager, D. Mueller, K. PassekKumericki, A. Schaefer, and M. Meskauskas, arXiv: 1105.0899.

[50] G. D. Cates, C. W. de Jager, S. Riordan, and B. Wojtsekhowski, Phys. Rev. Lett. 106, 252003 (2011).

[51] M. Schindler and S. Scherer, Eur. Phys. J. A 32, 429 (2007).

[52] T. Gorringe and H. W. Fearing, Rev. Mod. Phys. 76, 31 (2003).

[53] S. Ahmad, H. Honkanen, S. Liuti, and S. K. Taneja, Phys. Rev. D 75, 094003 (2007).

[54] S. Ahmad, H. Honkanen, S. Liuti, and S. K. Taneja, Eur. Phys. J. C 63, 407 (2009). 